\title{
The limit behavior of a family of variational multiscale problems
}

\author{
Margarida Baía and Irene Fonseca
}

February 15, 2007

\begin{abstract}
$\Gamma$-convergence techniques combined with techniques of 2 -scale convergence are used to give a characterization of the behavior as $\varepsilon$ goes to zero of a family of integral functionals defined on $L^{p}\left(\Omega ; \mathbb{R}^{d}\right)$ by

$$
\mathcal{I}_{\varepsilon}(u):= \begin{cases}\int_{\Omega} f\left(x, \frac{x}{\varepsilon}, \nabla u(x)\right) d x & \text { if } u \in W^{1, p}\left(\Omega ; \mathbb{R}^{d}\right), \\ \infty & \text { otherwise, }\end{cases}
$$

under periodicity (and nonconvexity) hypothesis, standard $p$-coercivity and $p$-growth conditions with $p>1$. Uniform continuity with respect to the $x$ variable, as it is customary in the existing literature, is not required.
\end{abstract}

Keywords: integral functionals, periodic integrands, $\Gamma$-convergence, 2-scale convergence, quasiconvexity, equi-integrability

MSC $2000(\mathcal{A M S}): 35 E 99,35 M 10,49 J 45,74 G 65$

\section{Introduction and main result}

The analysis of the limiting behavior of ordinary or partial differential (systems of) equations with oscillating and periodic coefficients was initiated using asymptotic expansions (see Bensoussan, Lions and Papanicolau [9], Jikov, Kozlov and Oleinik [39] and Sanchez-Palencia [52]), and it evolved toward more general situations through the concepts of $G$-convergence introduced by Spagnolo (see [53]), $H$-convergence due to Murat and Tartar (see [48] and [54]), $\Gamma$-convergence due to De Giorgi (see [27] and [29]), and of 2-scale convergence introduced by Nguetseng (see [42], [49] and [50]), further developed by Allaire and Briane (see [2] and [3]) and generalized by many other authors.

From a variational point of view the asymptotic analysis or homogenization of integral functionals, as it is referred in the literature, rests on the study of the equilibrium states, or minimizers, of a family of functionals of the type

$$
\mathcal{I}_{\varepsilon}(u)=\int_{\Omega} f_{\varepsilon}(x, \nabla u(x)) d x,
$$

where the functions $f_{\varepsilon}$ are increasingly oscillating in the first variable as the parameter $\varepsilon$ goes to zero, $\Omega$ is an open bounded set in $\mathbb{R}^{N}$ with $N \geqslant 1$, and $u$ is a scalar or vector-valued function in some Sobolev space. The understanding of the effective energy carried by these functionals leads to a "homogenized" functional $\mathcal{I}_{\text {hom }}$ such that a sequence of minimizers $u_{\varepsilon}$ of $\mathcal{I}_{\varepsilon}$ converges, as $\varepsilon$ goes to zero, to a limit $u$ 


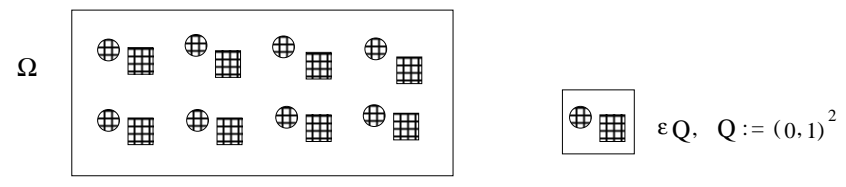

Figure 1: Typical example

that is a minimizer of the functional $\mathcal{I}_{\text {hom }}$. Hence $\mathcal{I}_{\text {hom }}$ captures the limiting behavior of equilibria, and a main quest in the Calculus of Variations is to obtain explicit characterizations of this functional, in particular to reach an integral representation formula of the type

$$
\mathcal{I}_{\text {hom }}=\int_{\Omega} f_{\text {hom }}(x, \nabla u(x)) d x
$$

where the effective energy density $f_{\text {hom }}$ is to be determined.

Our aim here is to characterize the behavior as $\varepsilon$ tends to zero of the family of functionals $\mathcal{I}_{\varepsilon}$ : $L^{p}\left(\Omega ; \mathbb{R}^{d}\right) \rightarrow[0, \infty]$ defined by

$$
\mathcal{I}_{\varepsilon}(u):= \begin{cases}\int_{\Omega} f\left(x, \frac{x}{\varepsilon}, \nabla u(x)\right) d x & \text { if } u \in W^{1, p}\left(\Omega ; \mathbb{R}^{d}\right), \\ \infty & \text { otherwise }\end{cases}
$$

under periodicity (and nonconvexity) hypothesis, $p$-coercivity and $p$-growth conditions with $p>1$. $\mathcal{I}_{\varepsilon}(u)$ can be interpreted as the energy under a deformation $u$ of an elastic body whose microstructure is periodic of period $\varepsilon$ (Figure. 1). We seek to approximate, in a $\Gamma$-convergence sense, the microscopic behavior of this kind of material by a macroscopic, or average, description. We combine a $\Gamma$-limit argument with techniques of 2 -scale convergence.

In the sequel given $\Omega$ an open bounded set in $\mathbb{R}^{N}$ with $N \geqslant 1$, we define $\mathcal{A}(\Omega)$ as the family of all its open subsets, and the notation $\Gamma\left(L^{p}(\Omega)\right.$ )-limit stands for the $\Gamma$-convergence with respect to the usual metric in $L^{p}\left(\Omega ; \mathbb{R}^{d}\right)(d \geqslant 1)$. The space $\mathbb{R}^{d \times N}$ will be identified with the set of real $d \times N$ matrices, $Q:=(0,1)^{N}$ is the unit cube in $\mathbb{R}^{N}$, and $\mathcal{L}^{N}$ or $|\cdot|$ stands for the usual Lebesgue measure in $\mathbb{R}^{N}$. Given $c \in \mathbb{R}$ we denote its integer part by $[c]$. Throughout the text $\beta$ represents a generic constant and $\varepsilon, \varepsilon_{n}$ are small positive numbers.

Functionals of the type (1.1) have been already studied in the $\Gamma$-convergence sense by many authors within the Sobolev and BV settings. In a Sobolev setting and for functionals of the form

$$
\int_{\Omega} f\left(\frac{x}{\varepsilon}, \nabla u(x)\right) d x
$$

we refer to Marcellini [44] and Carbone-Sbordone [21] for the scalar case, where it is assumed that $f=f(y, \xi)$ is Borel measurable, $Q$-periodic with respect to $y$ and convex with respect to $\xi$ (see also Cioranescu, Damlamian and De Arcangelis [23]). In the vectorial (and nonconvex) case we refer the reader to Müller [47] and Braides [12]. In [11] Braides studied functionals of the form

$$
\int_{\Omega} f\left(x, \frac{x}{\varepsilon}, u(x), \nabla u(x)\right) d x,
$$

for scalar-valued $u$ under the assumptions that the integrand $f=f(x, y, s, \xi)$ is convex in $\xi$, and that there exist a locally integrable function $b$ and a continuous positive real function $\omega$, with $\omega(0)=0$, such that 


$$
\left|f(x, y, s, \xi)-f\left(x^{\prime}, y, s^{\prime}, \xi\right)\right| \leqslant \omega\left(\left|x-x^{\prime}\right|+\left|s-s^{\prime}\right|\right)(b(y)+f(x, y, s, \xi))
$$

for all $x, x^{\prime}, y \in \mathbb{R}^{N}$ and $s, s^{\prime} \in \mathbb{R}^{d}$. In addition $f$ is assumed to be Borel measurable and $Q$-periodic with respect to $y$.

A sketch of the proof of an analogous result in the vectorial setting for $f=f(x, y, \xi)$ can be found in Braides and Defranceschi [15] (convex and nonconvex case) and also in Braides and Lukkassen [17] (convex case).

We refer also to Lukkassen [41], Braides and Lukkassen [17], Braides and Defranceschi [15], Fonseca and Zappale [38], Berlyand, Cioranescu and Golovaty [10], and Babadjian and Baía [5] for other multiscale problems in the $\Gamma$-convergence and Sobolev setting.

Using 2-scale convergence techniques we will substantially weaken the continuity hypothesis (1.2) required in the previous works without any convexity assumptions. We prove the following result.

Theorem 1.1. Let $f: \Omega \times \mathbb{R}^{N} \times \mathbb{R}^{d \times N} \rightarrow \mathbb{R}$ be a function such that

$\left(H_{1}\right) f(\cdot, y, \cdot)$ is continuous in $\Omega \times \mathbb{R}^{d \times N}$ for a.e. $y \in \mathbb{R}^{N}$;

$\left(H_{2}\right) f(x, \cdot, \xi)$ is measurable for all $x \in \Omega$ and for all $\xi \in \mathbb{R}^{d \times N}$;

$\left(H_{3}\right) f(x, \cdot, \xi)$ is Q-periodic for all $x \in \Omega$ and for all $\xi \in \mathbb{R}^{d \times N}$;

$\left(H_{4}\right)$ there exist a real number $p>1$ and a constant $\alpha>0$ such that

$$
\frac{|\xi|^{p}}{\alpha}-\alpha \leqslant f(x, y, \xi) \leqslant \alpha\left(1+|\xi|^{p}\right),
$$

for all $x \in \Omega$, for a.e. $y \in \mathbb{R}^{N}$ and for all $\xi \in \mathbb{R}^{d \times N}$.

If $u \in L^{p}\left(\Omega ; \mathbb{R}^{d}\right)$ then

$$
\mathcal{I}_{\mathrm{hom}}(u):=\Gamma\left(L^{p}(\Omega)\right)-\lim _{\varepsilon \rightarrow 0} \mathcal{I}_{\varepsilon}(u)= \begin{cases}\int_{\Omega} f_{\mathrm{hom}}(x, \nabla u(x)) d x & \text { if } u \in W^{1, p}\left(\Omega ; \mathbb{R}^{d}\right), \\ \infty & \text { otherwise }\end{cases}
$$

where

$$
f_{\mathrm{hom}}(x, \xi):=\lim _{T \rightarrow \infty} \inf _{\phi}\left\{\frac{1}{T^{N}} \int_{(0, T)^{N}} f(x, y, \xi+\nabla \phi(y)) d y, \phi \in W_{0}^{1, p}\left((0, T)^{N} ; \mathbb{R}^{d}\right)\right\}
$$

for all $x \in \Omega$ and all $\xi \in \mathbb{R}^{d \times N}$. Moreover, $f_{\text {hom }}$ is a Carathéodory function, satisfies similar p-coercivity and p-growth conditions to those of $f$, and $f_{\mathrm{hom}}(x, \cdot)$ is quasiconvex for all $x \in \Omega$.

As for quasiconvex envelopes, there are very few explicit examples of homogenized densities in the literature. A classical explicit derivation of the function $f_{\text {hom }}$ for elliptic operators in the homogeneous case, that is, for integrands $f$ that do not depend on the variable $x$, can be found in De Giorgi and Spagnolo [31] (see also the book of Dal Maso [25] and references therein).

The proof of Theorem 1.1 uses the direct method of $\Gamma$-convergence. The existence of $\Gamma$-converging (sub)sequences rests on the integral representation theorem of Buttazzo and Dal Maso (Theorem 2.10), and arguments of two-scale convergence are used to derive an upper bound for the integrand of this functional. To get the other bound we invoke the fact that, under hypotheses $\left(H_{1}\right)-\left(H_{4}\right), f$ is "uniformly continuous up to a small error". The argument rests on the Scorza Dragoni Theorem (Theorem 2.8), the 
periodicity of $f$ on $Q$, and the Decomposition Lemma (Theorem 2.20) that allows us to select minimizing sequences with $p$-equi-integrable gradients.

Two more remarks are worthy of note. First, it can be shown that for all $x \in \Omega$ and for all $\xi \in \mathbb{R}^{d \times N}$

$$
f_{\text {hom }}(x, \xi)=\inf _{T \in \mathbb{N}} \inf _{\phi}\left\{\frac{1}{T^{N}} \int_{(0, T)^{N}} f(x, y, \xi+\nabla \phi(y)) d y, \phi \in W_{0}^{1, p}\left((0, T)^{N} ; \mathbb{R}^{d}\right)\right\}
$$

and

$$
f_{\text {hom }}(x, \xi)=\inf _{T \in \mathbb{N}} \inf _{\phi}\left\{\frac{1}{T^{N}} \int_{(0, T)^{N}} f(x, y, \xi+\nabla \phi(y)) d y, \phi \in W_{\text {per }}^{1, p}\left((0, T)^{N} ; \mathbb{R}^{d}\right)\right\}
$$

(see [47] and [15]; see also Lemma 3.3). Secondly, we observe that under the additional hypothesis that $f(x, y, \cdot)$ is convex for all $x$ and a.e. $y,(1.5)$ or equivalently (1.6) simplify to read

$$
f_{\text {hom }}(x, \xi)=\inf _{\phi}\left\{\int_{Q} f(x, y, \xi+\nabla \phi(y)) d y, \phi \in W_{\text {per }}^{1, p}\left(Q ; \mathbb{R}^{d}\right)\right\} .
$$

Identity (1.7) asserts that for convex integrands it is sufficient to consider variations which are periodic in one cell $Q$, while for nonconvex integrands $f$ it is necessary to consider variations which are periodic over an infinite ensemble of cells. As noted by Müller, (1.7) hold for scalar $u$ without assuming any convexity hypothesis on $f$, and do not hold in the general vector-valued nonconvex case (see Section 4 in $[47])$. Moreover, by hypothesis $\left(H_{1}\right)$, the p-growth condition in $\left(H_{4}\right)$ and by a variant of the Dominated Convergence Theorem (see e.g. Theorem 4 in section 1.3 of Evans and Gariepy [34]) (1.4), (1.5)-(1.7) hold if the admissible test functions are taken in any smooth dense subset of $W_{0}^{1, p}\left((0, T)^{N} ; \mathbb{R}^{d}\right)$ and $W_{\text {per }}^{1, p}\left((0, T)^{N} ; \mathbb{R}^{d}\right)$, respectively.

The fundamental property of $\Gamma$-convergence, and its main link to other homogenization techniques, is that under certain compactness properties approximate minimizers of $\mathcal{I}_{\varepsilon}$ converge to a minimizer of the limiting functional $\mathcal{I}_{\text {hom }}$. This is made precise in the following corollary of Theorem 1.1.

Corollary 1.2. The functional $\mathcal{I}_{\text {hom }}$ has a minimum on $V_{\varphi}=\left\{u \in W^{1, p}\left(\Omega ; \mathbb{R}^{d}\right): u-\varphi \in W_{0}^{1, p}\left(\Omega ; \mathbb{R}^{d}\right)\right\}$ and

$$
\min _{u \in V_{\varphi}} \mathcal{I}_{\text {hom }}(u)=\lim _{\varepsilon \rightarrow 0} \inf _{u \in V_{\varphi}} \mathcal{I}_{\varepsilon}(u) .
$$

Moreover, given $\varepsilon_{n} \rightarrow 0$ and $\left\{u_{n}\right\}_{n} \subset V_{\varphi}$ such that

$$
\lim _{n \rightarrow \infty} \mathcal{I}_{\varepsilon_{n}}\left(u_{n}\right)=\min _{u \in V_{\varphi}} \mathcal{I}_{\text {hom }}(u),
$$

then (up to a subsequence) $\left\{u_{n}\right\}_{n} W^{1, p}$-weakly converges to a minimum of $\mathcal{I}_{\text {hom }}$ on $V_{\varphi}$.

We note that if $\Omega$ is assumed to be Lipschitz then the $\Gamma$-limit of the previous functionals for $u \in$ $W^{1, p}\left(\Omega ; \mathbb{R}^{d}\right)$ would be the same if the weak $W^{1, p}$-topology had been considered in place of the strong $L^{p}$-topology. For $p=1$ our argument fails to characterize this $\Gamma$-limit for $u \in W^{1,1}\left(\Omega ; \mathbb{R}^{d}\right)$, either with the strong $L^{1}$-topology or with the weak $W^{1,1}$-topology, since sequences whose gradients are bounded in $L^{1}$ (see (H4)) are not necessarily compact in $W^{1,1}$, as the argument carried out in Step 2 of the proof of Proposition 4.6 requires. These sequences are relatively compact only in the space of functions of bounded variation. The homogenization of functionals of linear growth in the framework of $\Gamma$-convergence and in the space of (special)bounded variation functions has been considered, among others, by Bouchitté [18], Braides and Chiadò Piat [14] and Carbone, Cioranescu, De Arcangelis and Gaudiello (see [22] and references therein) in the convex case; in the nonconvex case it has been treated by De Arcangelis and Gargiulo [4] and Bouchitté, Fonseca and Mascarenhas [19]. 
We remark that an analogous result to that of Theorem 1.1 holds if we assume the integrand $f=$ $f(x, y, \xi)$ to be measurable in $x$, continuous with respect to the pair $(y, \xi)$, and $Q$-periodic as a function of the variable $y$ (see Section 5). This case was presented in detail in Baía and Fonseca [6]. Recently, in an independent work, Pedregal [51] prove Theorem 1.1 in the scalar and convex case using Young measures techniques (see also Barchiesi [8]).

The overall plan of this work in the ensuing sections will be as follows: Section 2 collects the main definitions and auxiliary results used in the proof of Theorem 1.1. In Section 3 we record the properties of $f_{\text {hom }}$ for later use. These properties can be deduced from previous works but we present here alternative proofs for the sake of completeness. The proof of Theorem 1.1 is given in Section 4, and finally in Section 5 we present a similar result under a different set of hypotheses.

\section{Preliminaries}

The purpose of this section is to give a brief overview of concepts and results that are used throughout this work. All these results are stated without proofs as they can be readily found in the references given below.

\section{$2.1 \quad \Gamma$-convergence of a family of functionals}

We start by recalling De Giorgi's notion of $\Gamma$-convergence and some of its basic properties (see De Giorgi and dal Maso [27] and De Giorgi and Franzoni [29]). We refer to Braides [13] and Dal Maso [25] for a comprehensive treatment and bibliography on the subject.

Throughout this subsection $(X, d)$ denotes a metric space.

Definition 2.1. ( $\Gamma$-convergence of a sequence of functionals) Let $\left\{\mathcal{I}_{n}\right\}_{n}$ be a sequence of functionals defined on $X$ with values in $\overline{\mathbb{R}}$. The functional $\mathcal{I}: X \rightarrow \overline{\mathbb{R}}$ is said to be the $\Gamma$-liminf (resp. $\Gamma$-lim sup) of $\left\{\mathcal{I}_{n}\right\}_{n}$ with respect to the metric $d$ if for every $u \in X$

$$
\mathcal{I}(u)=\inf _{\left\{u_{n}\right\}}\left\{\liminf _{n \rightarrow \infty} \mathcal{I}_{n}\left(u_{n}\right): u_{n} \in X, u_{n} \rightarrow u \text { in } X\right\} \text { (resp. } \limsup _{n \rightarrow \infty} \text {. }
$$

In this case we write

$$
\mathcal{I}=\Gamma-\liminf _{n \rightarrow \infty} \mathcal{I}_{n} \quad\left(\operatorname{resp} . \quad \mathcal{I}=\Gamma-\limsup _{n \rightarrow \infty} \mathcal{I}_{n}\right) .
$$

Moreover, the functional $\mathcal{I}$ is said to be the $\Gamma$-lim of $\left\{\mathcal{I}_{n}\right\}_{n}$ if

$$
\mathcal{I}=\Gamma-\liminf _{n \rightarrow \infty} \mathcal{I}_{n}=\Gamma-\limsup _{n \rightarrow \infty} \mathcal{I}_{n},
$$

and in this case we write

$$
\mathcal{I}=\Gamma-\lim _{n \rightarrow \infty} \mathcal{I}_{n}
$$

For every $\varepsilon>0$ let $\mathcal{I}_{\varepsilon}$ be a functional over $X$ with values on $\overline{\mathbb{R}}, \mathcal{I}_{\varepsilon}: X \rightarrow \overline{\mathbb{R}}$.

Definition 2.2. ( $\Gamma$-convergence of a family of functionals)

A functional $\mathcal{I}: X \rightarrow \overline{\mathbb{R}}$ is said to be the $\Gamma$ - $\lim \inf \left(\right.$ resp. $\Gamma$-limsup or $\Gamma$-lim) of $\left\{\mathcal{I}_{\varepsilon}\right\}_{\varepsilon}$ with respect to the metric $d$, as $\varepsilon \rightarrow 0$, if for every sequence $\varepsilon_{n} \rightarrow 0$

$$
\mathcal{I}=\Gamma-\liminf _{n \rightarrow \infty} \mathcal{I}_{\varepsilon_{n}} \quad\left(\operatorname{resp} . \quad \mathcal{I}=\Gamma-\limsup _{n \rightarrow \infty} \mathcal{I}_{\varepsilon_{n}} \text { or } \mathcal{I}=\Gamma-\lim _{n \rightarrow \infty} \mathcal{I}_{\varepsilon_{n}}\right),
$$

and we write 


$$
\mathcal{I}=\Gamma-\liminf _{\varepsilon \rightarrow 0} \mathcal{I}_{\varepsilon} \quad\left(\operatorname{resp} . \quad \mathcal{I}=\Gamma-\limsup _{\varepsilon \rightarrow 0} \mathcal{I}_{\varepsilon} \text { or } \mathcal{I}=\Gamma-\lim _{\varepsilon \rightarrow 0} \mathcal{I}_{\varepsilon}\right)
$$

The next result states that a metric space $(X, d)$ satisfies the Urysohn property with respect to $\Gamma$ convergence.

Proposition 2.3. Given $\mathcal{I}: X \rightarrow \overline{\mathbb{R}}$ and $\varepsilon_{n} \rightarrow 0, \mathcal{I}=\Gamma-\lim _{n \rightarrow \infty} \mathcal{I}_{\varepsilon_{n}}$ if and only if for every subsequence

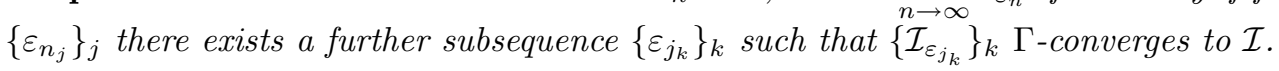

If, in addition, $(X, d)$ is a separable metric space then the following compactness property hold.

Theorem 2.4. Each sequence $\varepsilon_{n} \rightarrow 0$ has a subsequence $\left\{\varepsilon_{n_{j}}\right\}_{j}$ such that $\Gamma_{j \rightarrow \infty} \lim _{j \rightarrow \infty} \mathcal{I}_{\varepsilon_{n_{j}}}$ exists.

Proposition 2.5. If $\mathcal{I}=\Gamma-\liminf _{\varepsilon \rightarrow 0} \mathcal{I}_{\varepsilon}$ (or $\Gamma-\limsup _{\varepsilon \rightarrow 0}$ ) then $\mathcal{I}$ is lower semicontinuous (with respect to the metric d). Consequently, if $\mathcal{I}=\Gamma-\lim _{\varepsilon \rightarrow 0} \mathcal{I}_{\varepsilon}$ then $\mathcal{I}$ is lower semicontinuous.

Definition 2.6. A family of functionals $\left\{\mathcal{I}_{\varepsilon}\right\}_{\varepsilon}$ is said to be equi-coercive if for every real number $\lambda$ there exists a compact set $K_{\lambda}$ in $X$ such that for each sequence $\varepsilon_{n} \rightarrow 0$,

$$
\left\{u \in X: \mathcal{I}_{\varepsilon_{n}}(u) \leqslant \lambda\right\} \subseteq K_{\lambda} \text { for every } n \in \mathbb{N} .
$$

As mentioned before, one of the most important properties of $\Gamma$-convergence is that under appropriate compactness properties it implies the convergence of (almost) minimizers of a family of equi-coercive functionals to the minimum of the limiting functional. Precisely, we have the following result.

Theorem 2.7. (Fundamental Theorem of $\Gamma$-convergence) If $\left\{\mathcal{I}_{\varepsilon}\right\}_{\varepsilon}$ is a family of equi-coercive functionals on $X$ and if

$$
\mathcal{I}=\Gamma-\lim _{\varepsilon \rightarrow 0} \mathcal{I}_{\varepsilon}
$$

then the functional $\mathcal{I}$ has a minimum on $X$ and

$$
\min _{u \in X} \mathcal{I}(u)=\lim _{\varepsilon \rightarrow 0} \inf _{u \in X} \mathcal{I}_{\varepsilon}(u)
$$

Moreover, given $\varepsilon_{n} \rightarrow 0$ and $\left\{u_{n}\right\}_{n}$ a converging sequence such that

$$
\lim _{n \rightarrow \infty} \mathcal{I}_{\varepsilon_{n}}\left(u_{n}\right)=\lim _{n \rightarrow \infty} \inf _{u \in X} \mathcal{I}_{\varepsilon_{n}}(u),
$$

then its limit is a minimum point for $\mathcal{I}$ on $X$.

If (2.1) holds then $\left\{u_{n}\right\}_{n}$ is said to be a sequence of almost-minimizers for $\mathcal{I}$.

\subsection{Quasiconvex functions}

We recall that a Borel measurable function $f: \mathbb{R}^{d \times N} \rightarrow \mathbb{R}$ is said to be quasiconvex at a point $x \in \mathbb{R}^{d \times N}$ if

$$
f(x) \leqslant \int_{\Omega} f(x+\nabla \phi(y)) d y
$$

for every $\phi \in W_{0}^{1, \infty}\left(\Omega ; \mathbb{R}^{d}\right)$ and for every open bounded set $\Omega \subset \mathbb{R}^{N}$ with $\mathcal{L}^{N}(\partial \Omega)=0$, where $W_{0}^{1, \infty}\left(\Omega ; \mathbb{R}^{d}\right)$ is the space of Lipschitz functions in $\Omega$ with values on $\mathbb{R}^{d}$ and zero trace on $\partial \Omega$. The function $f$ is said 
to be quasiconvex if it is quasiconvex at any $x \in \mathbb{R}^{d \times N}$ (see Morrey [46]). As it is known, if $1 \leqslant p<\infty$ and if $f: \mathbb{R}^{d \times N} \rightarrow \mathbb{R}$ is quasiconvex and there exists $\gamma>0$ such that

$$
0 \leqslant f(\xi) \leqslant \gamma\left(1+|\xi|^{p}\right)
$$

for all $\xi \in \mathbb{R}^{d \times N}$, then the $p$-Lipschitz condition

$$
|f(x)-f(y)| \leqslant \beta\left(1+|x|^{p-1}+|y|^{p-1}\right)|x-y|
$$

holds for all $x, y \in \mathbb{R}^{d \times N}$, and some $\beta>0$ (see Marcellini [45]).

\subsection{Carathéodory functions}

Given $\Omega$ an open subset of $\mathbb{R}^{N}, N \geqslant 1$, and $B$ a Borel set of $\mathbb{R}^{l}, l \geqslant 1$, a function $f: \Omega \times B \rightarrow \overline{\mathbb{R}}$ is said to be a Carathéodory integrand if

i) $x \mapsto f(x, \xi)$ is measurable for every $\xi \in B$,

ii) $\xi \mapsto f(x, \xi)$ is continuous for almost all $x \in \Omega$.

In this work we deal with Carathéodory integrands where $l=N+(d \times N)$. We will use the following characterization.

Theorem 2.8. (Scorza-Dragoni Theorem) (see Ekeland and Teman [33]) Let $\Omega \subset \mathbb{R}^{N}, N \geqslant 1$, be an open set. A function $f: \Omega \times \mathbb{R}^{l} \rightarrow \mathbb{R}, l \geqslant 1$, is Carathéodory if and only if given a compact set $K \subset \Omega$ and a positive number $\varepsilon$, there exists a compact set $K_{\varepsilon} \subset K$ such that $\mathcal{L}^{N}\left(K \backslash K_{\varepsilon}\right) \leqslant \varepsilon$ and the restriction of $f$ to $K_{\varepsilon} \times \mathbb{R}^{l}$ is continuous.

The following result shows that every Carathéodory integrand is (equivalent to) a Borel function.

Proposition 2.9. (see Proposition 3.3 in Braides and Defranceschi [15] or Ekeland and Teman [33]) Let $\Omega \subset \mathbb{R}^{N}, N \geqslant 1$, be an open set, and let $B$ be a Borel set of $\mathbb{R}^{l}, l \geqslant 1$. Every Carathéodory integrand $f: \Omega \times B \rightarrow \mathbb{R}$ is (equivalent to) a Borel function, that is there exists a Borel function $g: \Omega \times B \rightarrow \mathbb{R}$ such that $f(x, \cdot)=g(x, \cdot)$ for a.e. $x \in \Omega$.

\subsection{An integral representation theorem for functionals defined over Sobolev spaces}

In this section we recall an integral representation theorem for local functionals depending on Sobolev functions and on open sets obtained by Buttazzo and Dal Maso. It provides abstract conditions under which a functional $\mathcal{I}$ admits an integral representation of the form

$$
\mathcal{I}(u, A)=\int_{A} f(x, \nabla u(x)) d x
$$

for some Carathéodory integrand $f$ (see Theorem 1.1 in [20] and references therein).

Theorem 2.10. Let $\Omega$ be an open subset of $\mathbb{R}^{N}, N \geqslant 1$. Let $\mathcal{I}: W^{1, p}\left(\Omega ; \mathbb{R}^{d}\right) \times \mathcal{A}(\Omega) \rightarrow \mathbb{R}$, with $d \geqslant 1$ and $1 \leqslant p \leqslant \infty$, where $\mathcal{A}(\Omega)$ is the set of open subsets of $\Omega$, satisfy the following properties

i) $\mathcal{I}$ is local on $\mathcal{A}(\Omega)$, i.e. $\mathcal{I}(u, A)=\mathcal{I}(v, A)$ whenever $A \in \mathcal{A}(\Omega), u, v \in W^{1, p}\left(\Omega ; \mathbb{R}^{d}\right)$ and $u=v$ a.e. on $A$;

ii) $\mathcal{I}$ is a measure on $\mathcal{A}(\Omega)$, i.e. for every $u \in W^{1, p}\left(\Omega ; \mathbb{R}^{d}\right)$ the set function $\mathcal{I}(u,$.$) is the restriction to$ $\mathcal{A}(\Omega)$ of a finite Radon measure; 
iii) I satisfies a growth condition of order $p$, i.e. if $p<\infty$ then there exist $a \in L^{1}(\Omega)$ and $b \geqslant 0$ such that for every $A \in \mathcal{A}(\Omega)$ and every $u \in W^{1, p}\left(\Omega ; \mathbb{R}^{d}\right)$,

$$
|\mathcal{I}(u, A)| \leqslant \int_{A}\left[a(x)+b|\nabla u|^{p}\right] d x
$$

and if $p=\infty$ then for every $r \geqslant 0$ there exists $a_{r} \in L^{1}(\Omega)$ such that

$$
|\mathcal{I}(u, A)| \leqslant \int_{A} a_{r}(x) d x
$$

for every $A \in \mathcal{A}(\Omega)$ and every $u \in W^{1, \infty}\left(\Omega ; \mathbb{R}^{d}\right)$ with $|\nabla u| \leqslant r$ a.e. in $A$;

iv) $\mathcal{I}$ is translation invariant, i.e. for every $A \in \mathcal{A}(\Omega), u \in W^{1, p}\left(\Omega ; \mathbb{R}^{d}\right), c \in \mathbb{R}^{d}$,

$$
\mathcal{I}(u+c, A)=\mathcal{I}(u, A) ;
$$

v) for every $A \in \mathcal{A}(\Omega)$, the function $\mathcal{I}(\cdot, A)$ is s.w.l.s.c on $W^{1, p}$ (s.w*.l.s.c if $\left.p=\infty\right)$.

Then, there exists a function $f: \Omega \times \mathbb{R}^{d \times N} \rightarrow \mathbb{R}$ such that

a) for every $A \in \mathcal{A}(\Omega)$ and every $u \in W^{1, p}\left(\Omega ; \mathbb{R}^{d}\right)$ the integral representation formula holds

$$
\mathcal{I}(u, A)=\int_{A} f(x, \nabla u(x)) d x
$$

b) $f$ is a Carathéodory integrand;

c) $f(x, z)$ satisfies a growth condition of order $p$, that is, when $p<\infty$ there exist $d \in L^{1}(\Omega)$ and $\beta \geqslant 0$ such that

$$
|f(x, z)| \leqslant d(x)+\beta|z|^{p},
$$

for a.e. $x \in \Omega$ and for all $z \in \mathbb{R}^{d \times N}$, and when $p=\infty$ for every $r \geqslant 0$ there exists $d_{r} \in L^{1}(\Omega)$ such that

$$
|f(x, z)| \leqslant d_{r}(x)
$$

for a.e. $x \in \Omega$ and for all $z \in \mathbb{R}^{d \times N}$ with $|z| \leqslant r$.

\section{Remark 2.11.}

i) Conditions a), b), c) imply i), ii), iii), iv) but not v). Nevertheless, the integral representation theorem does not hold if we drop hypothesis v) (see examples in Buttazzo and Dal Maso [20]).

ii) Conditions a), b), c) and v) imply that for a.e. $x \in \Omega$ the function $\xi \mapsto f(x, \xi)$ is quasiconvex (see Statment II.5 in Acerbi and Fusco [1]). 


\subsection{Sufficient conditions for a set function to be a Radon measure}

The following lemma provides sufficient conditions for a set function $\Pi: \mathcal{A}(X) \rightarrow[0, \infty)$ to be the restriction of a Radon measure to $\mathcal{A}(X)$, where $\mathcal{A}(X)$ is the set of open subsets of a topological space $X$. It is close in spirit to De Giorgi-Letta's criterion (see [30]) and it is of importance to apply the Direct Method of $\Gamma$-convergence as well as for the use of relaxation methods that strongly rely on the structure of Radon measures.

Lemma 2.12. (see Fonseca and Malý [36]; also Fonseca and Leoni [35]) Let $X$ be a locally compact Hausdorff space, let $\Pi: \mathcal{A}(X) \rightarrow[0, \infty)$, and let $\mu$ be a finite Radon measure $\mu$ on $X$ satisfying

i) (nested-subadditivity) $\Pi(D) \leq \Pi(D \backslash \bar{B})+\Pi(C)$ for all $B, C, D \in \mathcal{A}(X)$ with $B \subset \subset C \subset D$;

ii) Given $D \in \mathcal{A}(X)$, for all $\epsilon>0$ there exists $D_{\varepsilon} \in \mathcal{A}(X)$ such that $D_{\varepsilon} \subset \subset D$ and $\Pi\left(D \backslash \bar{D}_{\varepsilon}\right) \leq \varepsilon$;

iii) $\Pi(X) \geq \mu(X)$;

iv) $\Pi(D) \leq \mu(\bar{D})$ for all $D \in \mathcal{A}(X)$.

Then $\Pi=\left.\mu\right|_{\mathcal{A}(X)}$.

\subsection{The notion of 2-scale convergence}

In this section we present in a schematic way the main properties of two-scale convergence introduced by Nguetseng [49] (see [42] and also [50]) and further developed by Allaire and Briane (see [3] and Lukkassen, Nguetseng and Wall [42]).

Definition 2.13. (Periodic function) A function $f: \mathbb{R}^{N} \rightarrow \mathbb{R}$, with $N \geqslant 1$, is

i) $Q$ - periodic if $f(\cdot)=f\left(\cdot+l e_{i}\right)$ for all $l \in \mathbb{Z}$, where $\left\{e_{1}, \ldots, e_{N}\right\}$ is the canonical basis of $\mathbb{R}^{N}$;

ii) $k Q$-periodic (or $k$-periodic), with $k \in \mathbb{N}$, if $f(k \cdot)$ is $Q$-periodic.

We denote by $C_{\text {per }}(Q)$ the Banach space of all $Q$-periodic continuous functions defined in $\mathbb{R}^{N}$ with values in $\mathbb{R}$ endowed with the supremum norm, and by $W_{\text {per }}^{1, p}(k Q)$ the $W^{1, p}$-closure of all $k Q$-periodic and $C^{1}$-functions defined in $\mathbb{R}^{N}$ with values in $\mathbb{R}$ endowed with the $W^{1, p}$-norm.

Given $\Omega$ an open bounded subset of $\mathbb{R}^{N}$ and $1 \leqslant p<\infty$, we denote by $L^{p}\left(\Omega ; C_{\text {per }}(Q)\right)$ (resp. $\left.L^{p}\left(\Omega ; W_{\text {per }}^{1, p}(k Q)\right)\right)$ the space of all measurable functions $f: \Omega \rightarrow C_{\text {per }}(Q)$ (resp. $\left.f: \Omega \rightarrow W_{\text {per }}^{1, p}(k Q)\right)$ such that

$$
\begin{gathered}
\|f\|_{L^{p}\left(\Omega ; C_{\mathrm{per}}(Q)\right)}^{p}:=\int_{\Omega}\|f(x)\|_{C_{\mathrm{per}}(Q)}^{p} d x<\infty \\
\text { (resp. } \left.\|f\|_{L^{p}\left(\Omega ; W_{\mathrm{per}}^{1, p}(k Q)\right)}^{p}:=\int_{\Omega}\|f(x)\|_{W_{\mathrm{per}}^{1, p}(k Q)}^{p} d x<\infty\right)
\end{gathered}
$$

where

$$
\begin{gathered}
\|f(x)\|_{C_{\mathrm{per}}(Q)}:=\max _{y \in Q}|f(x, y)| \\
\text { (resp. } \left.\|f(x)\|_{W_{\mathrm{per}}^{1, p}(k Q)}^{p}:=\int_{k Q}|f(x, y)|^{p} d y+\int_{k Q}\left|\nabla_{y} f(x, y)\right|^{p} d y\right) .
\end{gathered}
$$

Clearly a function $f \in L^{p}\left(\Omega ; C_{\text {per }}(Q)\right)$ (resp. $\left.L^{p}\left(\Omega ; W_{\text {per }}^{1, p}(k Q)\right)\right)$ may be identified with the function defined on $\Omega \times \mathbb{R}^{N}$ via $f(x, y):=f(x)(y)\left(\nabla_{y} f\right.$ denotes its derivative with respect to the second argument $y)$. 
Similarly, $L_{\mathrm{per}}^{p}(Q ; C(\bar{\Omega}))$ stands for the space of functions $f=f(y)(x) \equiv f(y, x)$ that are measurable, $p$-summable, and $Q$-periodic in $y$, with values in the Banach space of continuous functions on $\bar{\Omega}$, with

$$
\|f\|_{L_{\mathrm{per}}^{p}(Q ; C(\bar{\Omega}))}^{p}:=\int_{Q}\|f(y)\|_{C(\bar{\Omega})}^{p} d y
$$

and

$$
\|f(y)\|_{C(\bar{\Omega})}:=\max _{x \in \bar{\Omega}}|f(y, x)| .
$$

Generalized versions of the Riemann-Lebesgue Lemma hold for functions in $L^{p}\left(\Omega ; C_{\mathrm{per}}(Q)\right)$ and in $L_{\mathrm{per}}^{p}(Q ; C(\bar{\Omega})), p \geqslant 1$.

Lemma 2.14. (see Lemma 5.2 in Allaire [2] and Theorem 3 in Lukkassen, Nguetseng and Wall [42]; see also Bensoussan, Lions and Papanicolaou [9] and Donato [32]) Let $f \in L^{p}\left(\Omega ; C_{\mathrm{per}}(Q)\right)$ and let $\left\{\varepsilon_{n}\right\}_{n}$ be a sequence of positive numbers converging to zero. Then for every $n \in \mathbb{N}$ the function $f\left(\cdot, \dot{\overline{\varepsilon_{n}}}\right)$ is measurable in $\Omega$,

$$
\left\|f\left(\cdot, \frac{\cdot}{\varepsilon_{n}}\right)\right\|_{L^{p}(\Omega)} \leqslant\|f\|_{L^{p}\left(\Omega ; C_{\mathrm{per}}(Q)\right)}
$$

and

$$
\lim _{n \rightarrow \infty} \int_{\Omega}\left|f\left(x, \frac{x}{\varepsilon_{n}}\right)\right|^{p} d x=\int_{\Omega} \int_{Q}|f(x, y)|^{p} d y d x .
$$

Lemma 2.15. (see Corollary 5.4 in Allaire [2]) Let $f \in L_{\mathrm{per}}^{p}(Q ; C(\bar{\Omega}))$ and let $\left\{\varepsilon_{n}\right\}_{n}$ be a sequence of positive numbers converging to zero. Then for every $n \in \mathbb{N}$ the function $f\left(\frac{\dot{\varepsilon_{n}}}{,}, \cdot\right)$ is measurable in $\Omega$,

$$
\left\|f\left(\frac{x}{\varepsilon_{n}}, x\right)\right\|_{L^{p}(\Omega)} \leqslant C\|f\|_{L_{\mathrm{per}}^{p}(Q ; C(\bar{\Omega}))}
$$

for some $C=C(\Omega)>0$, and

$$
\lim _{n \rightarrow \infty} \int_{\Omega}\left|f\left(\frac{x}{\varepsilon_{n}}, x\right)\right|^{p} d x=\int_{\Omega} \int_{Q}|f(y, x)|^{p} d y d x .
$$

Let $p$ and $q$ be real numbers such that $1<p<\infty$ and $\frac{1}{p}+\frac{1}{q}=1$, and let $\left\{\varepsilon_{n}\right\}_{n}$ be a sequence of positive numbers converging to zero.

Definition 2.16. (Two-scale convergence) $A$ sequence of functions $\left\{f_{n}\right\}_{n}$ in $L^{p}(\Omega)$ is said to two-scale converge to a limit $f \in L^{p}(\Omega \times Q)$, and we write $f_{n} \stackrel{2 s}{\rightarrow} f$, if

$$
\int_{\Omega} f_{n}(x) \phi\left(x, \frac{x}{\varepsilon_{n}}\right) d x \rightarrow \int_{\Omega} \int_{Q} f(x, y) \phi(x, y) d y d x,
$$

as $n \rightarrow \infty$, for all $\phi \in L^{q}\left(\Omega ; C_{\mathrm{per}}(Q)\right)$.

Lemma 2.17. (see e.g. Lukkassen, Nguetseng and Wall [42]) Let $\left\{f_{n}\right\}_{n} \subset L^{p}(\Omega)$ be such that $f_{n} \stackrel{2 s}{\rightarrow}$. Then

$$
\int_{\Omega} f_{n}(x) \phi\left(\frac{x}{\varepsilon_{n}}, x\right) d x \rightarrow \int_{\Omega} \int_{Q} f(x, y) \phi(y, x) d y d x,
$$

as $n \rightarrow \infty$, for all $\phi \in L_{\mathrm{per}}^{q}(Q ; C(\bar{\Omega}))$. 
Lemma 2.18. (see e.g. Lukkassen, Nguetseng and Wall [42]) Every sequence $\left\{f_{n}\right\}_{n}$ bounded in $L^{p}(\Omega)$ admits a subsequence (still denoted by $\left\{f_{n}\right\}_{n}$ ) such that $f_{n} \stackrel{2 s}{\rightarrow} f$ for some $f \in L^{p}(\Omega \times Q$ ).

For sequences weakly convergent in $W^{1, p}(\Omega)$ the following compactness result holds.

Theorem 2.19. (see Allaire [2] or Nguetseng [49]) Let $\left\{f_{n}\right\}_{n}$ be a sequence weakly convergent to a function $f$ in $W^{1, p}(\Omega)$. Then $f_{n} \stackrel{2 s}{f}$, and there exist a subsequence (still denoted by $\left\{f_{n}\right\}_{n}$ ) and $f_{1} \in L^{p}\left(\Omega ; W_{\mathrm{per}}^{1, p}(Q)\right)$ such that

$$
\nabla f_{n} \stackrel{2 s}{\rightarrow} \nabla f+\nabla_{y} f_{1}
$$

\subsection{The Decomposition Lemma}

We recall that a sequence of functions $\left\{u_{n}\right\} \subset L^{1}(\Omega)$ is said to be equi-integrable if for all $\varepsilon>0$ there exists $\delta>0$ such that

$$
\sup _{n \in \mathbb{N}} \int_{A}\left|u_{n}\right| d x<\varepsilon
$$

whenever $A \subset \Omega$ with $\mathcal{L}^{N}(A)<\delta$.

As a consequence of the next theorem, each sequence with bounded gradients in $L^{p}$, for $1<p<\infty$, admits a subsequence that can be decomposed as a sum of a sequence with $p$-equi-integrable gradients and a remainder that converges to zero in measure. This property turns out to be an important tool for the asymptotic analysis of integral functionals relying on localization arguments.

Theorem 2.20. (Decomposition Lemma) (see Fonseca and Leoni [35]; see also Fonseca, Müller and Pedregal [37] and Kristensen [43]) Assume that $\partial \Omega$ is Lipschitz, let $1<p<\infty$ and let $u_{n} \rightarrow v_{0}$ in $W^{1, p}\left(\Omega ; \mathbb{R}^{d}\right)$. Then there exist a subsequence $\left\{u_{n_{k}}\right\}_{k}$ of $\left\{u_{n}\right\}_{n}$ and a sequence $\left\{v_{k}\right\}_{k} \subset W^{1, \infty}\left(\mathbb{R}^{N} ; \mathbb{R}^{d}\right)$ such that

i) $v_{k} \rightarrow v_{0}$ in $W^{1, p}\left(\Omega ; \mathbb{R}^{d}\right)$,

ii) $v_{k}=v_{0}$ in a neighborhood of $\partial \Omega$,

iii) $\left\{\nabla v_{k}\right\}_{k}$ is p-equi-integrable,

iv) $\lim _{k \rightarrow \infty} \mathcal{L}^{N}\left(\left\{x \in \Omega: v_{k}(x) \neq u_{n_{k}}(x)\right\}\right)=0$.

\section{Properties of $f_{\text {hom }}$}

In this section we turn our attention to the main properties of the function $f_{\text {hom }}$ defined in (1.4). By hypothesis $\left(H_{4}\right)$ replacing $f$ by $f+\alpha$ we may assume throughout that $f$ is nonnegative.

We start by showing that the limit in (1.4) is well defined. This fact follows as a consequence of the next lemma, whose argument is analogous to that used in Bouchitté, Fonseca and Mascarenhas [19] and relies on Lemma 6.1 presented in Appendix.

Lemma 3.1. Let $f: \Omega \times \mathbb{R}^{N} \times \mathbb{R}^{d \times N} \rightarrow \mathbb{R}$ be a function such that

$\left(H_{1}^{\prime}\right) f(x, y, \cdot)$ is continuous in $\mathbb{R}^{d \times N}$ for all $x \in \Omega$ and for a.e. $y \in \mathbb{R}^{N}$,

and hypotheses $\left(H_{2}\right),\left(H_{3}\right)$ and $\left(H_{4}\right)$ hold. Then for all $\xi \in \mathbb{R}^{d \times N}$ there exists

$$
\lim _{T \rightarrow \infty} \inf _{\phi}\left\{\frac{1}{T^{N}} \int_{(0, T)^{N}} f(x, y, \xi+\nabla \phi(y)) d y, \phi \in W_{0}^{1, p}\left((0, T)^{N} ; \mathbb{R}^{d}\right)\right\} .
$$


Proof. (see also Braides and Defranceschi [15]) Let $(x, \xi) \in \Omega \times \mathbb{R}^{d \times N}$ and let

$$
S(A):=\inf _{\phi}\left\{\int_{A} f(x, y, \xi+\nabla \phi(y)) d y: \phi \in W_{0}^{1, p}\left(A ; \mathbb{R}^{d}\right)\right\}
$$

for $A \in \mathcal{A}\left(\mathbb{R}^{N}\right)$. Under the assumptions on $f$ the function $S: \mathcal{A}\left(\mathbb{R}^{N}\right) \rightarrow \mathbb{R}^{+}$satisfies the hypotheses of Lemma 6.1 with $T=\mathbb{Z}^{N}$ and $M=1$. Hence we conclude that the limit

$$
\lim _{T \rightarrow \infty} \frac{S\left((0, T)^{N}\right)}{T^{N}}
$$

or, equivalently, (3.1) exists.

In particular, if $f$ satisfies $\left(H_{1}\right)-\left(H_{4}\right)$ the conclusion of Lemma 3.1 holds. We want to show that under these hypotheses $f_{\text {hom }}$ is a continuous function. We start by showing that if $f$ satisfies hypotheses $\left(H_{1}^{\prime}\right)$ and $\left(H_{2}\right)-\left(H_{4}\right)$, then $f_{\text {hom }}(x, \cdot)$ is continuous for all $x \in \Omega$. This task would be greatly simplified if $f$ would satisfy a $p$-Lipschitz condition of the form (2.2). As quasiconvex functions under hypothesis $\left(H_{4}\right)$ satisfy inequality (2.2), the first step will be to verify that $f_{\text {hom }}=(\mathcal{Q} f)_{\text {hom }}$ where $\mathcal{Q} f: \Omega \times \mathbb{R}^{N} \times \mathbb{R}^{d \times N} \rightarrow \mathbb{R}$ denotes the usual quasiconvexification of $f$ with respect to the last variable $\xi$, and which is known to be quasiconvex in this last variable (see Dacorogna [24]). We recall that

$$
\mathcal{Q} f(x, y, \xi)=\inf _{\phi}\left\{\int_{Q} f(x, y, \xi+\nabla \phi(z)) d z: \phi \in W_{0}^{1, p}\left(Q ; \mathbb{R}^{d}\right)\right\}
$$

for all $(x, y, \xi) \in \Omega \times \mathbb{R}^{N} \times \mathbb{R}^{d \times N}$ (see Dacorogna [24] and Ball and Murat [7]) and that, consequently, $\mathcal{Q} f$ satisfies conditions $\left(H_{3}\right)$ and $\left(H_{4}\right)$. The following properties of $\mathcal{Q} f$ are of interest for the argument that follows.

Lemma 3.2. Let $f$ satisfy hypotheses $\left(H_{1}^{\prime}\right)$ and $\left(H_{2}\right)-\left(H_{4}\right)$. We have that

i) $\mathcal{Q} f(x, \cdot, \cdot)$ is a Carathéodory function for all $x \in \Omega$;

ii) $(\mathcal{Q} f)_{\text {hom }}(x, \xi)=f_{\text {hom }}(x, \xi)$ for all $(x, \xi) \in \Omega \times \mathbb{R}^{d \times N}$.

Proof. i) Let $(x, \xi) \in \Omega \times \mathbb{R}^{d \times N}$. We can write

$$
\mathcal{Q} f(x, y, \xi)=\inf _{\phi \in \mathcal{S}_{T}} g_{\phi}(y)
$$

where

$$
g_{\phi}(y):=\int_{Q} f(x, y, \xi+\nabla \phi(z)) d z
$$

and $\mathcal{S}_{T}$ is a countable subset of $C_{c}^{\infty}\left((0, T)^{N} ; \mathbb{R}^{d}\right)$ dense in $W_{0}^{1, p}\left((0, T)^{N} ; \mathbb{R}^{d}\right)$. By Tonelli's Theorem the functions $g_{\phi}$ are measurable, and so is $\mathcal{Q} f(x, \cdot, \xi)$ as the infimum of a countable family of measurable functions. The upper semicontinuity of $\mathcal{Q} f(x, y, \cdot)$ for all $x \in \Omega$ and for a.e. $y \in \mathbb{R}^{N}$ follows from (3.2), hypotheses $\left(H_{1}\right)$ and $\left(H_{4}\right)$. Its lower semicontinuity can be obtained using an argument analogous to that of Lemma 4.3 in Dal Maso, Fonseca, Leoni and Morini [26].

ii) As a consequence of i) and of Lemma 3.1 we remark that

$$
\begin{aligned}
(\mathcal{Q} f)_{\mathrm{hom}}(x, \xi): & =\liminf _{T \rightarrow \infty} \inf _{\phi}\left\{\frac{1}{T^{N}} \int_{(0, T)^{N}} \mathcal{Q} f(x, y, \xi+\nabla \phi(y)) d y, \phi \in W_{0}^{1, p}\left((0, T)^{N} ; \mathbb{R}^{d}\right)\right\} \\
& =\lim _{T \rightarrow \infty} \inf _{\phi}\left\{\frac{1}{T^{N}} \int_{(0, T)^{N}} \mathcal{Q} f(x, y, \xi+\nabla \phi(y)) d y, \phi \in W_{0}^{1, p}\left((0, T)^{N} ; \mathbb{R}^{d}\right)\right\}
\end{aligned}
$$


for all $(x, \xi) \in \Omega \times \mathbb{R}^{d \times N}$.

Let $(x, \xi) \in \Omega \times \mathbb{R}^{d \times N}$. Obviously $f_{\text {hom }}(x, \xi) \geqslant(\mathcal{Q} f)_{\text {hom }}(x, \xi)$. Let us prove the converse inequality. Let $n \in \mathbb{N}$ and let $T_{n} \in \mathbb{N}$ and $\phi_{n} \in W_{0}^{1, p}\left(\left(0, T_{n}\right)^{N} ; \mathbb{R}^{d}\right)$ be such that

$$
(\mathcal{Q} f)_{\mathrm{hom}}(x, \xi)+\frac{1}{n} \geqslant \frac{1}{T_{n}^{N}} \int_{\left(0, T_{n}\right)^{N}} \mathcal{Q} f\left(x, y ; \xi+\nabla \phi_{n}(y)\right) d y .
$$

Thus

$$
(\mathcal{Q} f)_{\text {hom }}(x, \xi) \geqslant \limsup _{n \rightarrow \infty} \frac{1}{T_{n}^{N}} \int_{\left(0, T_{n}\right)^{N}} \mathcal{Q} f\left(x, y ; \xi+\nabla \phi_{n}(y)\right) d y .
$$

To compare (3.3) with $f_{\text {hom }}(x, \xi)$ we apply the Relaxation Theorem of Acerbi and Fusco (Statement III.7 in [1]) and the Decomposition Lemma (Theorem 2.20). As a consequence of the first result, for every $n$ fixed there exists a sequence $\left\{\phi_{n, k}\right\}_{k} \subset W^{1, p}\left(\left(0, T_{n}\right)^{N} ; \mathbb{R}^{d}\right)$ such that $\phi_{n, k} \underset{k}{\rightarrow} \phi_{n}$ in $W^{1, p}\left(\left(0, T_{n}\right)^{N} ; \mathbb{R}^{d}\right)$ and

$$
\frac{1}{T_{n}^{N}} \int_{\left(0, T_{n}\right)^{N}} \mathcal{Q} f\left(x, y ; \xi+\nabla \phi_{n}(y)\right) d y=\lim _{k \rightarrow \infty} \frac{1}{T_{n}^{N}} \int_{\left(0, T_{n}\right)^{N}} f\left(x, y ; \xi+\nabla \phi_{n, k}(y)\right) d y .
$$

By Theorem 2.20 we can now find a subsequence (still denoted by $\left\{\phi_{n, k}\right\}_{k}$ ) and a sequence $\left\{\psi_{n, k}\right\}_{k} \subset$ $W_{0}^{1, \infty}\left(\mathbb{R}^{N} ; \mathbb{R}^{d}\right)$ such that $\psi_{n, k} \rightarrow \phi_{n}$ in $W^{1, p}\left(\left(0, T_{n}\right)^{N} ; \mathbb{R}^{d}\right)$ with

$$
\left\{\left|\nabla \psi_{n, k}\right|^{p}\right\} \quad \text { equi-integrable }
$$

and

$$
\mathcal{L}^{N}\left\{y \in\left(0, T_{n}\right)^{N}: \psi_{n, k}(y) \neq \phi_{n, k}(y)\right\} \underset{k \rightarrow \infty}{\longrightarrow} 0 .
$$

As $f$ is nonnegative, by $(3.5),(3.6)$ and $\left(H_{4}\right)$

$$
\begin{aligned}
& \lim _{k \rightarrow \infty} \frac{1}{T_{n}^{N}} \int_{\left(0, T_{n}\right)^{N}} f\left(x, y ; \xi+\nabla \phi_{n, k}(y)\right) d y \\
\geqslant & \limsup _{k \rightarrow \infty} \frac{1}{T_{n}^{N}} \int_{\left\{y \in\left(0, T_{n}\right)^{N}: \psi_{n, k}(y)=\phi_{n, k}(y)\right\}} f\left(x, y ; \xi+\nabla \psi_{n, k}(y)\right) d y \\
= & \limsup _{k \rightarrow \infty} \frac{1}{T_{n}^{N}} \int_{\left(0, T_{n}\right)^{N}} f\left(x, y ; \xi+\nabla \psi_{n, k}(y)\right) d y .
\end{aligned}
$$

Thus from (3.3), (3.4) and (3.7)

$$
(\mathcal{Q} f)_{\text {hom }}(x, \xi) \geqslant \limsup _{n \rightarrow \infty} \limsup _{k \rightarrow \infty} \frac{1}{T_{n}^{N}} \int_{\left(0, T_{n}\right)^{N}} f\left(x, y, \xi+\nabla \psi_{n, k}(y)\right) d y \geqslant f_{\text {hom }}(x, \xi) .
$$

We note now the following result.

Lemma 3.3. Let $f$ satisfy $\left(H_{1}^{\prime}\right)$ and $\left(H_{2}\right)-\left(H_{4}\right)$, and let $\bar{f}_{\text {hom }}, \hat{f}_{\text {hom }}: \Omega \times \mathbb{R}^{d \times N} \rightarrow[0, \infty)$ be defined by

$$
\bar{f}_{\text {hom }}(x, \xi):=\inf _{T \in \mathbb{N}} \inf _{\phi}\left\{\frac{1}{T^{N}} \int_{(0, T)^{N}} f(x, y, \xi+\nabla \phi(y)) d y, \phi \in W_{0}^{1, p}\left((0, T)^{N} ; \mathbb{R}^{d}\right)\right\}
$$

and

$$
\hat{f}_{\text {hom }}(x, \xi):=\inf _{T \in \mathbb{N}} \inf _{\phi}\left\{\frac{1}{T^{N}} \int_{(0, T)^{N}} f(x, y, \xi+\nabla \phi(y)) d y, \phi \in W_{\text {per }}^{1, p}\left((0, T)^{N} ; \mathbb{R}^{d}\right)\right\},
$$

for all $(x, \xi) \in \Omega \times \mathbb{R}^{d \times N}$. Then $f_{\text {hom }}=\bar{f}_{\text {hom }}=\hat{f}_{\text {hom }}$. 
Proof. Let $(x, \xi) \in \Omega \times \mathbb{R}^{d \times N}$. We first show that $f_{\text {hom }}(x, \xi)=\bar{f}_{\text {hom }}(x, \xi)$. It is clear that $f_{\text {hom }}(x, \xi) \geqslant$ $\bar{f}_{\text {hom }}(x, \xi)$. To prove the other inequality, fixed $\delta>0$ and let $T \in \mathbb{N}, \varphi \in W_{0}^{1, p}\left((0, T)^{N} ; \mathbb{R}^{d}\right)$, be such that

$$
\bar{f}_{\text {hom }}(x, \xi)+\delta \geqslant \frac{1}{T^{N}} \int_{(0, T)^{N}} f(x, y, \xi+\nabla \varphi(y)) d y .
$$

Extend $\varphi$ periodically to $\mathbb{R}^{N}$ with period $T$. Using Riemann-Lebesgue's Lemma, and by $\left(H_{3}\right)$,

$$
\begin{aligned}
\frac{1}{T^{N}} \int_{(0, T)^{N}} f(x, y, \xi+\nabla \varphi(y)) d y & =\lim _{\varepsilon \rightarrow 0} \frac{1}{T^{N}} \int_{(0, T)^{N}} f\left(x, \frac{y}{\varepsilon}, \xi+\nabla \varphi\left(\frac{y}{\varepsilon}\right)\right) d y \\
& =\lim _{\varepsilon \rightarrow 0} \frac{\varepsilon^{N}}{T^{N}} \int_{\left(0, \frac{T}{\varepsilon}\right)^{N}} f\left(x, z, \xi+\nabla \theta_{\varepsilon}(z)\right) d z,
\end{aligned}
$$

where $\theta_{\varepsilon}(z):=\frac{1}{\varepsilon} \varphi(\varepsilon z) \in W_{0}^{1, p}\left(\left(0, \frac{T}{\varepsilon}\right)^{N} ; \mathbb{R}^{d}\right)$. Therefore, from (3.8) and (3.9) we have

$$
\begin{aligned}
\bar{f}_{\text {hom }}(x, \xi)+\delta & \geqslant \lim _{\varepsilon \rightarrow 0} \inf _{\theta}\left\{\frac{\varepsilon^{N}}{T^{N}} \int_{\left(0, \frac{T}{\varepsilon}\right)^{N}} f(x, z, \xi+\nabla \theta(z)) d z, \theta \in W_{0}^{1, p}\left(\left(0, \frac{T}{\varepsilon}\right)^{N} ; \mathbb{R}^{d}\right)\right\} \\
& =f_{\text {hom }}(x, \xi) .
\end{aligned}
$$

Letting $\delta \rightarrow 0$ we conclude that

$$
\bar{f}_{\text {hom }}(x, \xi) \geqslant f_{\text {hom }}(x, \xi) .
$$

Finally we show that $\bar{f}_{\text {hom }}(x, \xi)=\hat{f}_{\text {hom }}(x, \xi)$ (see also Braides [15] and Müller [47] for an alternative justification). It is clear that $\bar{f}_{\text {hom }}(x, \xi) \geqslant \hat{f}_{\text {hom }}(x, \xi)$.

To verify the opposite inequality, fix $\delta>0$ and find $T \in \mathbb{N}, \varphi \in W_{\text {per }}^{1, p}\left((0, T)^{N} ; \mathbb{R}^{d}\right)$, such that

$$
\hat{f}_{\text {hom }}(x, \xi)+\delta \geqslant \frac{1}{T^{N}} \int_{(0, T)^{N}} f(x, y, \xi+\nabla \varphi(y)) d y .
$$

By hypothesis $\left(H_{3}\right)$ the function $f(x, \cdot, \xi+\nabla \varphi(\cdot))$ is $(0, T)^{N}$-periodic, and thus

$$
\begin{aligned}
\frac{1}{T^{N}} \int_{(0, T)^{N}} f(x, y, \xi+\nabla \varphi(y)) d y & =\lim _{\varepsilon \rightarrow 0} \int_{Q} f\left(x, \frac{y}{\varepsilon}, \xi+\nabla \varphi\left(\frac{y}{\varepsilon}\right)\right) d y \\
& =\lim _{\varepsilon \rightarrow 0} \int_{Q} f\left(x, \frac{y}{\varepsilon}, \xi+\nabla \psi_{\varepsilon}(y)\right) d y
\end{aligned}
$$

where $\psi_{\varepsilon}(y):=\varepsilon \varphi\left(\frac{y}{\varepsilon}\right)$. For each $\varepsilon>0$ define

$$
Q_{\varepsilon}:=\{y \in Q: \operatorname{dist}(y, \partial Q)>\varepsilon\} .
$$

Let $\theta_{\varepsilon} \in C_{c}^{\infty}(Q,[0,1])$ be such that $\theta_{\varepsilon} \equiv 1$ in $Q_{\varepsilon}$ and

$$
\left\|\nabla \theta_{\varepsilon}\right\|_{L^{\infty}} \leqslant \beta \varepsilon^{-1}
$$

for some $\beta>0$. Then

$$
\limsup _{\varepsilon \rightarrow 0} \int_{Q} f\left(x, \frac{y}{\varepsilon}, \xi+\nabla\left(\theta_{\varepsilon} \psi_{\varepsilon}\right)(y)\right) d y=\limsup _{\varepsilon \rightarrow 0} \int_{Q_{\varepsilon}} f\left(x, \frac{y}{\varepsilon}, \xi+\nabla \psi_{\varepsilon}(y)\right) d y,
$$


since by the $p$-growth condition in $\left(H_{4}\right)$ and $(3.12)$ we have

$$
\begin{aligned}
& \int_{Q \backslash Q_{\varepsilon}} f\left(x, \frac{y}{\varepsilon}, \xi+\nabla\left(\theta_{\varepsilon} \psi_{\varepsilon}\right)(y)\right) d y \\
\leqslant & \beta \int_{Q \backslash Q_{\varepsilon}}\left(1+|\xi|^{p}+\left|\nabla \psi_{\varepsilon}(y)\right|^{p}+\varepsilon^{-p}\left|\psi_{\varepsilon}(y)\right|^{p}\right) d y \\
= & \beta\left(\left|Q \backslash Q_{\varepsilon}\right|+\int_{Q \backslash Q_{\varepsilon}}\left|\nabla \varphi\left(\frac{y}{\varepsilon}\right)\right|^{p} d y+\int_{Q \backslash Q_{\varepsilon}}\left|\varphi\left(\frac{y}{\varepsilon}\right)\right|^{p} d y\right) \rightarrow 0 .
\end{aligned}
$$

Hence by (3.10)-(3.13), defining $\phi_{\varepsilon}(y):=\frac{1}{\varepsilon}\left(\theta_{\varepsilon} \psi_{\varepsilon}\right)(\varepsilon y) \in W_{0}^{1, p}\left(\left(0, \frac{1}{\varepsilon}\right)^{N} ; \mathbb{R}^{N}\right)$, we obtain

$$
\begin{aligned}
\hat{f}_{\text {hom }}(x, \xi)+\delta & \geqslant \limsup _{\varepsilon \rightarrow 0} \int_{Q} f\left(x, \frac{y}{\varepsilon}, \xi+\nabla\left(\theta_{\varepsilon} \psi_{\varepsilon}\right)(y)\right) d y \\
& \geqslant \limsup _{\varepsilon \rightarrow 0} \varepsilon^{N} \int_{\left(0, \frac{1}{\varepsilon}\right)^{N}} f\left(x, y, \xi+\nabla \phi_{\varepsilon}(y)\right) d y \\
& \geqslant \bar{f}_{\text {hom }}(x, \xi) .
\end{aligned}
$$

Letting $\delta \rightarrow 0$ we conclude that $f_{\text {hom }}(x, \xi) \geqslant \bar{f}_{\text {hom }}(x, \xi)$.

We are now in a position to prove the continuity property of $f_{\text {hom }}$ with respect to its second variable.

Lemma 3.4. Let $f$ satisfies hypotheses $\left(H_{1}^{\prime}\right)$ and $\left(H_{2}\right)-\left(H_{4}\right)$. Then $f_{\mathrm{hom}}(x, \cdot)$ (or equivalently $\left.(\mathcal{Q} f)_{\mathrm{hom}}(x, \cdot)\right)$ is continuous for all $x \in \Omega$.

Proof. (see also Braides [15]) Fix $x \in \Omega$. Let $\xi \in \mathbb{R}^{d \times N}$ and $\xi_{n} \rightarrow \xi$ in $\mathbb{R}^{d \times N}$. We first establish that (upper semicontinuity)

$$
f_{\text {hom }}(x, \xi) \geqslant \limsup _{n \rightarrow \infty} f_{\text {hom }}\left(x, \xi_{n}\right) .
$$

Fixed $\delta>0$ and, in view of Lemma 3.3, choose $T \in \mathbb{N}$ and $\varphi \in W_{0}^{1, p}\left((0, T)^{N} ; \mathbb{R}^{d}\right)$ such that

$$
\begin{aligned}
f_{\text {hom }}(x, \xi)+\delta & \geqslant \frac{1}{T^{N}} \int_{(0, T)^{N}} f(x, y, \xi+\nabla \varphi(y)) d y \\
& =\frac{1}{T^{N}} \lim _{n \rightarrow \infty} \int_{(0, T)^{N}} f\left(x, y, \xi_{n}+\nabla \varphi(y)\right) d y \\
& \geqslant \limsup _{n \rightarrow \infty} f_{\text {hom }}\left(x, \xi_{n}\right),
\end{aligned}
$$

as a consequence of a variant of the Dominated Convergence Theorem. Letting $\delta \rightarrow 0$ we get (3.14).

We show now the converse inequality (lower semicontinuity), i.e.

$$
f_{\text {hom }}(x, \xi) \leqslant \liminf _{n \rightarrow \infty} f_{\text {hom }}\left(x, \xi_{n}\right) .
$$

Given $n \in \mathbb{N}$ consider $T_{n} \in \mathbb{N}$ and $\phi_{n} \in W_{0}^{1, p}\left(\left(0, T_{n}\right)^{N} ; \mathbb{R}^{d}\right)$ such that

$$
\begin{aligned}
f_{\text {hom }}\left(x, \xi_{n}\right)+\frac{1}{n} & \geqslant \frac{1}{T_{n}^{N}} \int_{\left(0, T_{n}\right)^{N}} f\left(x, y, \xi_{n}+\nabla \phi_{n}(y)\right) d y \\
& \geqslant \frac{1}{T_{n}^{N}} \int_{\left(0, T_{n}\right)^{N}} \mathcal{Q} f\left(x, y, \xi_{n}+\nabla \phi_{n}(y)\right) d y \\
& =\int_{Q} \mathcal{Q} f\left(x, T_{n} y, \xi_{n}+\nabla \phi_{n}\left(T_{n} y\right)\right) d y \\
& =\int_{Q} \mathcal{Q} f\left(x, T_{n} y, \xi_{n}+\nabla \psi_{n}(y)\right) d y
\end{aligned}
$$


where $\psi_{n}(y):=\frac{1}{T_{n}} \phi_{n}\left(T_{n} y\right), \psi_{n} \in W_{0}^{1, p}\left(Q ; \mathbb{R}^{d}\right)$. By the $p$-coervivity property of $\mathcal{Q} f$ the sequence $\left\{\left\|\nabla \psi_{n}\right\|_{L^{p}\left(Q ; \mathbb{R}^{d}\right)}\right\}$ is bounded. We write

$$
\begin{aligned}
& \int_{Q} \mathcal{Q} f\left(x, T_{n} y, \xi_{n}+\nabla \psi_{n}(y)\right) d y= \\
& \int_{Q} \mathcal{Q} f\left(x, T_{n} y, \xi_{n}+\nabla \psi_{n}(y)\right)-\mathcal{Q} f\left(x, T_{n} y, \xi+\nabla \psi_{n}(y)\right) d y \\
& +\int_{Q} \mathcal{Q} f\left(x, T_{n} y, \xi+\nabla \psi_{n}(y)\right) d y .
\end{aligned}
$$

We claim that that the term (3.17) goes to zero as $n$ goes to infinity. Using the $p$-Lipschitz condition (2.2) and Hölder Inequality we have

$$
\begin{aligned}
& \limsup _{n \rightarrow \infty} \int_{Q}\left|\mathcal{Q} f\left(x, T_{n} y, \xi_{n}+\nabla \psi_{n}(y)\right)-\mathcal{Q} f\left(x, T_{n} y, \xi+\nabla \psi_{n}(y)\right)\right| d y \\
& \leqslant \beta \limsup _{n \rightarrow \infty} \int_{Q}\left(1+\left|\xi_{n}+\nabla \psi_{n}(y)\right|^{p-1}+\left|\xi+\nabla \psi_{n}(y)\right|^{p-1}\right)\left|\xi_{n}-\xi\right| d y \\
& \leqslant \beta \limsup _{n \rightarrow \infty} \int_{Q}\left(1+\left|\nabla \psi_{n}(y)\right|^{p-1}\right)\left|\xi_{n}-\xi\right| d y \\
& \leqslant \beta \lim _{n \rightarrow \infty}\left|\xi_{n}-\xi\right|=0,
\end{aligned}
$$

which, together with (3.16), leads to

$$
f_{\mathrm{hom}}\left(x, \xi_{n}\right)+\frac{1}{n} \geqslant \limsup _{n \rightarrow \infty} \int_{Q} \mathcal{Q} f\left(x, T_{n} y, \xi+\nabla \psi_{n}(y)\right) d y \geqslant(\mathcal{Q} f)_{\mathrm{hom}}(x, \xi)=f_{\mathrm{hom}}(x, \xi),
$$

where in the last equality we used Lemma 3.2 ii). Inequality (3.15) follows by letting $n \rightarrow \infty$.

In particular if $f$ satisfies hypotheses $\left(H_{1}\right)-\left(H_{4}\right)$ then $f_{\text {hom }}(x, \cdot)$ is continuous for all $x \in \Omega$. We show now that under these conditions $f_{\text {hom }}(\cdot, \xi)$ is continuous for all $\xi \in \mathbb{R}^{d \times N}$.

Lemma 3.5. Let $f$ satisfies $\left(H_{1}\right)-\left(H_{4}\right)$. Then the function $f_{\text {hom }}(\cdot, \xi)$ is continuous for all $\xi \in \mathbb{R}^{d \times N}$.

Proof. Let $\xi \in \mathbb{R}^{d \times N}$. The upper semicontinuity of this function follows as a consequence of Lemma 3.3 by an argument analogous to that of Lemma 3.2. Let us see that $f_{\text {hom }}(\cdot, \xi)$ is lower semicontinuous. Let $x \in \mathbb{R}^{N}$ and $x_{n} \rightarrow x$. Let $n \in \mathbb{N}$ and let $T_{n} \in \mathbb{N}$ and $\varphi_{n} \in W_{0}^{1, p}(Q ; \mathbb{R})$ be such that

$$
f_{\mathrm{hom}}\left(x_{n}, \xi\right)+\frac{1}{n} \geqslant \int_{Q} f\left(x_{n}, T_{n} y, \xi+\nabla \varphi_{n}(y)\right) d y .
$$

Due to condition $\left(H_{4}\right)$ and by the Decomposition Lemma (Theorem 2.20) we may assume, without lost of generality, that $\left\{\left|\nabla \varphi_{n}\right|^{p}\right\}_{n}$ is equi-integrable. Let $a_{i, n} \in \mathbb{Z}^{N}, i \in\left\{1, \ldots,\left[T_{n}\right]^{N}\right\}$, be such that

$$
\bigcup_{i=1}^{\left[T_{n}\right]^{N}}\left(a_{i, n}+Q\right)=T_{n} Q
$$

and the cubes $a_{i, n}+Q$ are mutually disjoint. Then changing variables 


$$
\begin{aligned}
f_{\mathrm{hom}}\left(x_{n}, \xi\right)+\frac{1}{n} & \geqslant \frac{1}{T_{n}^{N}} \sum_{i=1}^{T_{n}^{N}} \int_{a_{i, n}+Q} f\left(x_{n}, y, \xi+\nabla \varphi_{n}\left(\frac{y}{T_{n}}\right)\right) d y \\
& =\frac{1}{T_{n}^{N}} \sum_{i=1}^{T_{n}^{N}} \int_{Q} f\left(x_{n}, y, \xi+\nabla \varphi_{n}\left(\frac{y+a_{i, n}}{T_{n}}\right)\right) d y
\end{aligned}
$$

due to the periodicity hypothesis $\left(H_{3}\right)$. Let $r>0$ be such that $\overline{B(x, r)} \subset \Omega$. Let $\theta_{r} \in C_{0}^{\infty}\left(\mathbb{R}^{N}\right)$ be such that $\theta_{r}=1$ in $B(x, r)$ and $\theta_{r}=0$ in $\mathbb{R}^{N} \backslash B(x, 2 r)$. Define $f_{r}:=f \theta_{r}$. By Scorza-Dragoni's Theorem (Theorem 2.8) applied to $f_{r}: \mathbb{R}^{N} \times Q \times \mathbb{R}^{d \times N} \rightarrow \mathbb{R}$, given $m \in \mathbb{N}$ let $K_{m} \subset \subset Q$ be a compact set with $\left|Q \backslash K_{m}\right| \leqslant 1 / m$ and such that $f_{r}: \mathbb{R}^{N} \times K_{m} \times \mathbb{R}^{d \times N} \rightarrow \mathbb{R}$ is continuous. Then $f_{r}: \overline{B(x, r)} \times K_{m} \times \overline{B(0, m)} \rightarrow \mathbb{R}$ is uniformly continuous. Let $n \in \mathbb{N}$ be such that $\left|x_{n}-x\right| \leqslant r$. For each $m \in \mathbb{N}$

$$
f_{\text {hom }}\left(x_{n}, \xi\right)+\frac{1}{n} \geqslant \frac{1}{T_{n}^{N}} \sum_{i=1}^{T_{n}^{N}} \int_{Q_{m, i, n}} f\left(x_{n}, y, \xi+\nabla \varphi_{n}\left(\frac{y+a_{i, n}}{T_{n}}\right)\right) d y
$$

where

$$
Q_{m, i, n}=\left\{y \in K_{m}:\left|\nabla \varphi_{n}\right|\left(\frac{y+a_{i}}{T_{n}}\right) \leqslant m\right\}
$$

We write

$$
\begin{gathered}
\int_{Q_{m, i, n}} f\left(x_{n}, y, \xi+\nabla \varphi_{n}\left(\frac{y+a_{i, n}}{T_{n}}\right)\right) d y \\
=\int_{Q_{m, i, n}}\left[f_{r}\left(x_{n}, y, \xi+\nabla \varphi_{n}\left(\frac{y+a_{i, n}}{T_{n}}\right)\right)-f_{r}\left(x, y, \xi+\nabla \varphi_{n}\left(\frac{y+a_{i, n}}{T_{n}}\right)\right)\right] d y \\
+\int_{Q_{m, i, n}} f\left(x, y, \xi+\nabla \varphi_{n}\left(\frac{y+a_{i, n}}{T_{n}}\right)\right) d y
\end{gathered}
$$

As $f_{r}: \overline{B(x, r)} \times K_{m} \times \overline{B(0, m)} \rightarrow \mathbb{R}$ is uniformly continuous, (3.21) goes to zero as $n \rightarrow \infty$. Thus 


$$
\begin{aligned}
\liminf _{n \rightarrow \infty} f_{\text {hom }}\left(x_{n}, \xi\right) & \geqslant \liminf _{m \rightarrow \infty} \liminf _{n \rightarrow \infty} \frac{1}{T_{n}^{N}} \sum_{i=1}^{T_{n}^{N}} \int_{Q_{m, i, n}} f\left(x, y, \xi+\nabla \varphi_{n}\left(\frac{y+a_{i, n}}{T_{n}}\right)\right) d y \\
& =\liminf _{m \rightarrow \infty} \liminf _{n \rightarrow \infty} \frac{1}{T_{n}^{N}} \sum_{i=1}^{T_{n}^{N}} \int_{a_{i, n}+Q_{m, i, n}} f\left(x, y-a_{i, n}, \xi+\nabla \varphi_{n}\left(\frac{y}{T_{n}}\right)\right) d y \\
& =\liminf _{m \rightarrow \infty} \liminf _{n \rightarrow \infty} \frac{1}{T_{n}^{N}} \sum_{i=1}^{T_{n}^{N}} \int_{a_{i, n}+Q_{m, i, n}} f\left(x, y, \xi+\nabla \varphi_{n}\left(\frac{y}{T_{n}}\right)\right) d y \\
& =\liminf _{m \rightarrow \infty} \liminf _{n \rightarrow \infty} \int_{\bigcup_{i=1}^{T N} \frac{\left(a_{i, n}+Q_{m, i, n}\right)}{T_{n}}} f\left(x, T_{n} y, \xi+\nabla \varphi_{n}(y)\right) d y
\end{aligned}
$$

by the periodicity condition $\left(H_{3}\right)$. Note that

$$
\liminf _{m \rightarrow \infty} \liminf _{n \rightarrow \infty} \int_{Q \backslash \bigcup_{i=1}^{T_{n}^{N}} \frac{\left(a_{i, n}+Q_{m, i, n}\right)}{T_{n}}} f\left(x, T_{n} y, \xi+\nabla \varphi_{n}(y)\right) d y=0 .
$$

Indeed, by hypothesis $\left(H_{4}\right)$

$$
\int_{Q \backslash \bigcup_{i=1}^{T_{n}^{N}} \frac{\left(a_{i, n}+Q_{m, i, n}\right)}{T_{n}}} f\left(x, T_{n} y, \xi+\nabla \varphi_{n}(y)\right) d y \leqslant \int_{Q \backslash \bigcup_{i=1}^{T_{n}^{N}} \frac{\left(a_{i, n}+Q_{m, i, n}\right)}{T_{n}}} C\left(1+\left|\nabla \varphi_{n}(y)\right|^{p}\right) d y .
$$

In addition,

$$
\lim _{m \rightarrow \infty} \lim _{n \rightarrow \infty}\left|Q \backslash \bigcup_{i=1}^{T_{n}^{N}} \frac{\left(a_{i, n}+Q_{m, i, n}\right)}{T_{n}^{N}}\right|=0
$$

since

$$
\begin{aligned}
\left|Q \backslash \bigcup_{i=1}^{T_{n}^{N}} \frac{\left(a_{i, n}+Q_{m, i, n}\right)}{T_{n}^{N}}\right| & \leqslant \sum_{i=1}^{T_{n}^{N}}\left|\frac{\left(a_{i, n}+Q\right)}{T_{n}} \backslash \frac{\left(a_{i, n}+Q_{m, i, n}\right)}{T_{n}}\right| \\
& =\frac{1}{T_{n}^{N}} \sum_{i=1}^{T_{n}^{N}}\left|Q \backslash Q_{m, i, n}\right| \\
& \leqslant \frac{1}{T_{n}^{N}} \sum_{i=1}^{T_{n}^{N}}\left[\left|Q \backslash K_{m}\right|+\left|K_{m} \backslash Q_{m, i, n}\right|\right]
\end{aligned}
$$

and 


$$
\begin{aligned}
\frac{1}{T_{n}^{N}} \sum_{i=1}^{T_{n}^{N}}\left[\left|Q \backslash K_{m}\right|+\left|K_{m} \backslash Q_{m, i, n}\right|\right] & \leqslant \frac{1}{m}+\frac{1}{m^{p}} \frac{1}{T_{n}^{N}} \sum_{i=1}^{T_{n}^{N}} \int_{K_{m}}\left|\nabla \varphi_{n}\right|^{p}\left(\frac{y+a_{i, n}}{T_{n}}\right) d y \\
& \leqslant \frac{1}{m}+\frac{1}{m^{p}} \frac{1}{T_{n}^{N}} \sum_{i=1}^{T_{n}^{N}} \int_{a_{i, n}+Q}\left|\nabla \varphi_{n}\right|^{p}\left(\frac{y}{T_{n}}\right) d y \\
& =\frac{1}{m}+\frac{1}{m^{p}} \frac{1}{T_{n}^{N}} \int_{T_{n} Q}\left|\nabla \varphi_{n}\right|^{p}\left(\frac{y}{T_{n}}\right) d y \\
& =\frac{1}{m}+\frac{1}{m^{p}} \int_{Q}\left|\nabla \varphi_{n}\right|^{p}(y) d y \\
& \leqslant \frac{1}{m}+\frac{\beta}{m^{p}} \rightarrow 0
\end{aligned}
$$

as $m \rightarrow \infty$, independently of $n$. Hence (3.23) holds by the equi-integrability property of $\left\{\nabla \varphi_{n}\right\}_{n}$ and (3.24). Then, by (3.22) we get that

$$
\liminf _{n \rightarrow \infty} f_{\text {hom }}\left(x_{n}, \xi\right) \geqslant \liminf _{n \rightarrow \infty} \int_{Q} f\left(x, T_{n} y, \xi+\nabla \varphi_{n}(y)\right) d y \geqslant f_{\text {hom }}(x, \xi),
$$

which asserts the claim.

Remark 3.6. We note that $f_{\text {hom }}$ satisfies growth and coercivity conditions similar to the ones of $f$ which, together with the continuity properties of $f_{\text {hom }}$, imply by standard arguments (approximation of $W^{1, p}$ by piecewise affine functions together with the invariance of the domain of $f_{\text {hom }}$ ) that this function is quasiconvex with respect to the last variable.

As a consequence of Lemmas 3.4 and 3.5, Remark 3.6 and of the p-Lipschitz condition (2.2) we conclude the following result.

Lemma 3.7. If $f$ satisfies $\left(H_{1}\right)-\left(H_{4}\right)$ then $f_{\text {hom }}$ is continuous.

We will show next that in the convex case it suffices to consider one cell period for the definition of $f_{\text {hom }}(1.4)$ (see also Braides and Defranceschi [15] or Müller [47] for alternative proofs). We define for all $(x, \xi) \in \Omega \times \mathbb{R}^{d \times N}$

$$
f_{\text {hom }}^{\#}(x, \xi)=\inf _{\phi}\left\{\int_{Q} f(x, y, \xi+\nabla \phi(y)) d y, \quad \phi \in W_{\text {per }}^{1, p}\left(Q ; \mathbb{R}^{d}\right)\right\} .
$$

Lemma 3.8. Assume in addition to the hypotheses $\left(H_{1}\right)-\left(H_{4}\right)$ on $f$ that $f(x, y, \cdot)$ is convex for all $x \in \Omega$ and for a.e. $y \in \mathbb{R}^{N}$. Then

$$
f_{\text {hom }}=f_{\text {hom }}^{\#} \text {. }
$$

Proof. We show that $f_{\text {hom }}=f_{\text {hom }}^{\#}$. Let $(x, \xi) \in \Omega \times \mathbb{R}^{d \times N}$. By Lemma $3.3 f_{\text {hom }}(x, \xi) \leqslant f^{\#}{ }_{\text {hom }}(x, \xi)$. To prove the opposite inequality, for each $n \in \mathbb{N}$ choose $T_{n} \in \mathbb{N}$ and a function $\phi_{n} \in W_{0}^{1, p}\left(\left(0, T_{n}\right)^{N} ; \mathbb{R}^{d}\right)$ such that 


$$
\begin{aligned}
f_{\text {hom }}(x, \xi)+\frac{1}{n} & \geqslant \frac{1}{T_{n}^{N}} \int_{\left(0, T_{n}\right)^{N}} f\left(x, y, \xi+\nabla \phi_{n}(y)\right) d y \\
& =\int_{Q} f\left(x, T_{n} y, \xi+\nabla \psi_{n}(y)\right) d y,
\end{aligned}
$$

where $\psi_{n}(y):=\frac{1}{T_{n}} \phi_{n}\left(T_{n} y\right), \psi_{n} \in W_{0}^{1, p}\left(Q ; \mathbb{R}^{d}\right)$. We note that by the $p$-growth condition in $\left(H_{4}\right)$ the sequence $\left\{\left\|\nabla \psi_{n}\right\|_{L^{p}\left(Q ; \mathbb{R}^{d}\right)}\right\}_{n}$ is bounded, and so is $\left\{\left\|\psi_{n}\right\|_{W^{1, p}\left(Q ; \mathbb{R}^{d}\right)}\right\}_{n}$ by Poincaré Inequality. Hence, there exists a subsequence (still denoted by $\left\{\psi_{n}\right\}_{n}$ ) such that

$$
\psi_{n} \stackrel{W^{1, p}}{\rightarrow} \psi
$$

for some $\psi=\psi(y) \in W_{0}^{1, p}\left(Q ; \mathbb{R}^{d}\right)$. As a consequence, by Theorem 2.19 and up to a subsequence

$$
\psi_{n} \stackrel{2 s}{\rightarrow} \psi
$$

and

$$
\nabla \psi_{n} \stackrel{2 s}{\rightarrow} \nabla \psi+\nabla_{z} \bar{\psi}
$$

for some $\bar{\psi}=\bar{\psi}(y, z) \in L^{p}\left(Q ; W_{\text {per }}^{1, p}\left(Q ; \mathbb{R}^{d}\right)\right)$. We divide the rest of the proof in two steps.

Step 1. We follow an argument of Allaire [2], assuming in addition that

$$
\left(H_{5}\right):\left\{\begin{array}{l}
\frac{\partial f}{\partial \eta}(x, y, \xi) \text { exists for all }(x, y, \xi) \in \Omega \times \mathbb{R}^{N} \times \mathbb{R}^{d \times N}, \\
\frac{\partial f}{\partial \xi}(x, \cdot, \cdot) \in L_{\mathrm{per}}^{q}\left(Q ; C\left(\mathbb{R}^{d \times N}\right)\right), \frac{1}{p}+\frac{1}{q}=1, \text { for all } x \in \Omega, \\
\left|\frac{\partial f}{\partial \eta}(x, y, \xi)\right|^{q} \leqslant \gamma\left(1+|\xi|^{p}\right), \gamma>0, \text { for all }(x, y, \xi) \in \Omega \times \mathbb{R}^{N} \times \mathbb{R}^{d \times N} .
\end{array}\right.
$$

Let $\varphi=\varphi(y, z) \in C_{c}^{\infty}\left(Q \times Q ; \mathbb{R}^{d \times N}\right)$ and extend $\varphi(y, \cdot) Q$-periodically to $\mathbb{R}^{N}$. Since $f$ is convex in the last variable then

$$
\begin{aligned}
\int_{Q} f\left(x, T_{n} y, \xi+\nabla \psi_{n}(y)\right) d y \geqslant & \int_{Q} \\
& f\left(x, T_{n} y, \xi+\varphi\left(y, T_{n} y\right)\right) d y \\
& +\int_{Q} \frac{\partial f}{\partial \xi}\left(x, T_{n} y, \xi+\varphi\left(y, T_{n} y\right)\right) \cdot\left(\nabla \psi_{n}(y)-\varphi\left(y, T_{n} y\right)\right) d y
\end{aligned}
$$

for each $n \in \mathbb{N}$. By Lemmas 2.15 and 2.17 we have

$$
\begin{aligned}
\liminf _{n \rightarrow \infty} \int_{Q} f\left(x, T_{n} y\right. & \left., \xi+\nabla \psi_{n}(y)\right) d y \geqslant \int_{Q}\left[\int_{Q} f(x, z, \xi+\varphi(y, z)) d z\right] d y \\
& +\int_{Q}\left[\int_{Q} \frac{\partial f}{\partial \xi}(x, z, \xi+\varphi(y, z)) \cdot\left(\nabla \psi(y)+\nabla_{z} \bar{\psi}(y, z)-\varphi(y, z)\right) d z\right] d y
\end{aligned}
$$

where we used the fact that

$$
(y, z) \mapsto \frac{\partial f}{\partial \xi}(x, z, \xi+\varphi(y, z)) \in L_{\mathrm{per}}^{q}(Q ; C(\bar{Q})) .
$$


Let now $\left\{\varphi_{k}\right\}_{k} \subset C_{c}^{\infty}\left(Q \times Q ; \mathbb{R}^{d \times N}\right)$ be a sequence convergent to $\nabla \psi+\nabla_{z} \bar{\psi}$ in $L^{p}\left(Q \times Q ; \mathbb{R}^{d \times N}\right)$. From (3.27) we have

$$
\begin{aligned}
\liminf _{n \rightarrow \infty} \int_{Q} f\left(x, T_{n} y, \xi\right. & \left.+\nabla \psi_{n}(y)\right) d y \geqslant \int_{Q}\left[\int_{Q} f\left(x, z, \xi+\varphi_{k}(y, z)\right) d z\right] d y \\
& +\int_{Q}\left[\int_{Q} \frac{\partial f}{\partial \xi}\left(x, z, \xi+\varphi_{k}(y, z)\right) \cdot\left(\nabla \psi(y)+\nabla_{z} \bar{\psi}(y, z)-\varphi_{k}(y, z)\right) d z\right] d y
\end{aligned}
$$

for every $k \in \mathbb{N}$, which, together with the growth conditions on $f$ and $\frac{\partial f}{\partial \xi}$, implies that

$$
\liminf _{n \rightarrow \infty} \int_{Q} f\left(x, T_{n} y, \xi+\nabla \psi_{n}(y)\right) d y \geqslant \int_{Q}\left[\int_{Q} f\left(x, z, \xi+\nabla \psi(y)+\nabla_{z} \bar{\psi}(y, z)\right) d z\right] d y .
$$

By Jensen's Inequality and Fubini's Theorem, for each $z \in Q$

$$
\begin{aligned}
\int_{Q} f\left(x, z, \xi+\nabla \psi(y)+\nabla_{z} \bar{\psi}(y, z)\right) d y & \geqslant f\left(x, z, \int_{Q}\left[\xi+\nabla \psi(y)+\nabla_{z} \bar{\psi}(y, z)\right] d y\right) \\
& =f\left(x, z, \xi+\nabla_{z}\left(\int_{Q} \bar{\psi}(y, z) d y\right)\right) .
\end{aligned}
$$

Thus by (3.26), (3.28), (3.29) and once more Fubini's Theorem,

$$
f_{\mathrm{hom}}(\xi) \geqslant \int_{Q} f\left(x, z, \xi+\nabla_{z}\left(\int_{Q} \bar{\psi}(y, z) d y\right)\right) d z \geqslant f_{\mathrm{hom}}^{\#}(\xi) .
$$

Step 2. Now we address the general case when $\left(H_{5}\right)$ may not be satisfied. For each $\varepsilon>0$ set $\zeta_{\varepsilon}(\eta):=$ $\frac{1}{\varepsilon^{N}} \zeta\left(\frac{\eta}{\varepsilon}\right)$ where $\zeta \in C^{\infty}\left(\mathbb{R}^{d \times N}\right)$ denotes the standard mollifier, that is,

$$
\zeta(\eta):= \begin{cases}\beta \exp \left(\frac{1}{|\eta|^{2}-1}\right) & \text { if }|\eta|<1, \\ 0 & \text { if }|\eta| \geqslant 1,\end{cases}
$$

and the constant $\beta$ is selected so that $\int_{\mathbb{R}^{d \times N}} \zeta(\eta) d \eta=1$. Let

$$
f_{\varepsilon}(x, y, \xi):=\int_{B(0, \varepsilon)} \zeta_{\varepsilon}(\eta) f(x, y, \xi-\eta) d \eta
$$

for all $\varepsilon>0$ and all $(x, y, \xi) \in \Omega \times \mathbb{R}^{N} \times \mathbb{R}^{d \times N}$. It is straightforward to show that $f_{\varepsilon}$ satisfies conditions $\left(H_{1}\right)-\left(H_{5}\right)$. Fixed $\delta>0$, by density let $T \in \mathbb{N}$ and $\psi \in W^{1, \infty}\left((0, T)^{N} ; \mathbb{R}^{d}\right)$ be such that

$$
f_{\mathrm{hom}}(x, \xi)+\delta \geqslant \frac{1}{T^{N}} \int_{(0, T)^{N}} f(x, y, \xi+\nabla \psi(y)) d y .
$$

Then 


$$
\begin{aligned}
f_{\text {hom }}(x, \xi)+\delta & \geqslant \lim _{\varepsilon \rightarrow 0} \frac{1}{T^{N}} \int_{(0, T)^{N}} f_{\varepsilon}(x, y, \xi+\nabla \psi(y)) d y \\
& \geqslant \limsup _{\varepsilon \rightarrow 0}\left(f_{\varepsilon}\right)_{\text {hom }}(x, \xi) \\
& =\limsup _{\varepsilon \rightarrow 0}\left(f_{\varepsilon}\right)_{\text {hom }}^{\#}(x, \xi) \\
& \geqslant f_{\text {hom }}^{\#}(x, \xi)
\end{aligned}
$$

where we used Step 1 to obtain the equality, and the fact that $f_{\varepsilon} \geqslant f$ in the last inequality. We remark that $f_{\varepsilon} \geqslant f$ as a consequence of the convexity of $f$ and Jensen's Inequality in Banach spaces (see e.g. Lemma 23.2 in Dal Maso [25]).

In the scalar case, that is when $d=1$, the identity (3.25) still holds independent of any convexity assumption on $f$ (see also Müller [47]). Precisely, we have the following result.

Corollary 3.9. Let $f: \Omega \times \mathbb{R}^{N} \times \mathbb{R}^{N} \rightarrow \mathbb{R}$ satisfy hypotheses $\left(H_{1}\right)-\left(H_{4}\right)$. Then

$$
f_{\text {hom }}=f^{\#}{ }_{\text {hom }} \text {. }
$$

Proof. Clearly $f_{\text {hom }} \leqslant f^{\#}$ hom. To see that $f_{\text {hom }} \geqslant f^{\#}$ hom we remark that an argument similar to that of Lemma 3.2 ii) yields

$$
f_{\text {hom }}^{\#}=(Q f)^{\#}{ }_{\text {hom }} \text {. }
$$

As $d=1$ we have that $Q f=C f$, hence $(Q f)^{\#}{ }_{\text {hom }}=(C f)^{\#}{ }_{\text {hom }}$, where $C f$ stands for the convex envelope of $f$, and by Lemma 3.8

$$
(C f)_{\mathrm{hom}}=(C f)^{\#}{ }_{\text {hom }} \text {. }
$$

Thus

$$
f_{\mathrm{hom}} \geqslant(C f)_{\mathrm{hom}}=(C f)^{\#}{ }_{\text {hom }}=(Q f)^{\#}{ }_{\text {hom }}=f^{\#}{ }_{\text {hom }} .
$$

\section{Proof of Theorem 1.1}

By hypothesis $\left(H_{4}\right)$ replacing $f$ by $f+\alpha$ we may assume throughout that $f$ is nonnegative. Due to the $p$-coercivity condition in $\left(H_{4}\right)$, to prove Theorem 1.1 it suffices to show that

$$
\Gamma\left(L^{p}(\Omega)\right)-\lim _{\varepsilon \rightarrow 0} \mathcal{I}_{\varepsilon}(u)=\int_{\Omega} f_{\text {hom }}(x, \nabla u(x)) d x,
$$

for all $u \in W^{1, p}\left(\Omega ; \mathbb{R}^{d}\right)$, where $f_{\text {hom }}$ is the function defined in (1.4), since

$$
\Gamma\left(L^{p}(\Omega)\right)-\lim _{\varepsilon \rightarrow 0} \mathcal{I}_{\varepsilon}(u)=\infty
$$

for all $u \in L^{p}\left(\Omega ; \mathbb{R}^{N}\right) \backslash W^{1, p}\left(\Omega ; \mathbb{R}^{d}\right)$. The idea behind the proof of identity (4.1) is to use the direct method of $\Gamma$ - convergence, first outlined by De Giorgi (see [28]; see also Dal Maso [25] and De Giorgi and Dal Maso [27]). Accordingly, we start by localizing the functionals $\mathcal{I}_{\varepsilon}$ in order to highlight their dependence on the domain of integration, that is, we consider a family of functionals $\mathcal{I}_{\varepsilon}: L^{p}\left(\Omega ; \mathbb{R}^{d}\right) \times \mathcal{A}(\Omega) \rightarrow[0, \infty]$ defined by 


$$
\mathcal{I}_{\varepsilon}(u ; A):= \begin{cases}\int_{A} f\left(x, \frac{x}{\varepsilon}, \nabla u(x)\right) d x & \text { if } u \in W^{1, p}\left(A ; \mathbb{R}^{d}\right), \\ \infty & \text { otherwise. }\end{cases}
$$

Our goal is to show that

$$
\Gamma\left(L^{p}(\Omega)\right)-\lim _{\varepsilon \rightarrow 0} \mathcal{I}_{\varepsilon}(u, A)=\int_{A} f_{\mathrm{hom}}(x, \nabla u(x)) d x,
$$

for all $u \in W^{1, p}\left(\Omega ; \mathbb{R}^{d}\right)$ and $A \in \mathcal{A}(\Omega)$. In particular (4.1) will follow by taking $A=\Omega$.

The next step toward the proof of Theorem 1.1 is to establish a compactness property that ensures the existence of $\Gamma$-converging subsequences of $\mathcal{I}_{\varepsilon}$.

Proposition 4.1. For every sequence $\left\{\varepsilon_{n}\right\}_{n}$ converging to zero there exists a further subsequence $\left\{\varepsilon_{n_{j}}\right\}_{j} \equiv$ $\left\{\varepsilon_{j}\right\}_{j}$ such that

$$
\Gamma\left(L^{p}(A)\right)-\lim _{j \rightarrow \infty} \mathcal{I}_{\varepsilon_{j}}(\cdot ; A)(u)=: \mathcal{I}^{\left\{\varepsilon_{j}\right\}}(u ; A)
$$

exists for all $u \in W^{1, p}\left(\Omega, \mathbb{R}^{d}\right)$ and all $A \in \mathcal{A}(\Omega)$.

The proof of this proposition follows an argument analog to the one used in Braides, Fonseca and Francfort [16], but for completeness we present it here.

Let $\mathcal{C}$ be a countable collection of open subsets of $\Omega$ such that for any $\delta>0$ and any $A \in \mathcal{A}(\Omega)$ there exists a finite union $C_{A}$ of disjoint elements of $\mathcal{C}$ satisfying

$$
\left\{\begin{array}{l}
\overline{C_{A}} \subset A, \\
\mathcal{L}^{N}(A) \leqslant \mathcal{L}^{N}\left(C_{A}\right)+\delta .
\end{array}\right.
$$

We may take $\mathcal{C}$ as the set of open squares with faces parallel to the axes, centered at points $x \in \Omega \cap \mathbb{Q}^{N}$ and with rational edge lengths. We denote by $\mathcal{R}$ the countable collection of all finite unions of elements of $\mathcal{C}$, i.e,

$$
\mathcal{R}=\left\{\bigcup_{i=1}^{k} C_{i}: k \in \mathbb{N}, C_{i} \in \mathcal{C}\right\}
$$

The next lemma is the starting point for the proof of Proposition 4.1.

Lemma 4.2. For every sequence $\left\{\varepsilon_{n}\right\}_{n}$ converging to zero there exists a further subsequence $\left\{\varepsilon_{n_{j}}\right\}_{j}$ (depending on $\mathcal{R}$ ) such that the $\Gamma$-limit

$$
\Gamma\left(L^{p}(C)\right)-\lim _{j \rightarrow \infty} \mathcal{I}_{\varepsilon_{n_{j}}}(\cdot ; C)(u)=: \mathcal{I}^{\left\{\varepsilon_{n_{j}}\right\}}(u ; C)
$$

exists for all $u \in W^{1, p}\left(\Omega, \mathbb{R}^{d}\right)$ and all $C \in \mathcal{R}$.

Proof. Let $C \in \mathcal{R}$. From Proposition 2.4 and as $L^{p}\left(\Omega ; \mathbb{R}^{d}\right)$ is separable, there exist a subsequence $\left\{\varepsilon_{n_{j}}\right\}_{j}$ (depending on $C$ ) such that the $\Gamma\left(L^{p}(C)\right.$ )-limit of $\mathcal{I}_{\varepsilon_{n_{j}}}(\cdot ; C)$ exists for all $u \in W^{1, p}\left(\Omega, \mathbb{R}^{d}\right)$. A diagonalization procedure yields a subsequence $\left\{\varepsilon_{n_{j}}\right\}_{j}$ (depending on $\mathcal{R}$ ) such that (4.3) holds.

Let now $\left\{\varepsilon_{n}\right\}_{n}$ be a fixed sequence of positive real numbers converging to zero and let $\left\{\varepsilon_{j}\right\}_{j}$ be a subsequence for which (4.3) holds. 
In order to prove that the $\Gamma$-limit in (4.2) exists for all $A \in \mathcal{A}(\Omega)$, it is crucial to establish the existence of recovering sequences for the $\Gamma$-limit in (4.3) with identical values on the boundaries of the elements of $\mathcal{R}$.

Lemma 4.3. Given $u \in W^{1, p}\left(\Omega ; \mathbb{R}^{d}\right)$ and $C \in \mathcal{R}$ there exists a sequence $\left\{w_{j}\right\} \subset W^{1, p}\left(C ; \mathbb{R}^{d}\right)$ such that

i) $w_{j} \rightarrow u$ strongly in $L^{p}\left(C ; \mathbb{R}^{d}\right)$;

ii) $\Gamma\left(L^{p}(C)\right)-\lim _{j \rightarrow \infty} \mathcal{I}_{\varepsilon_{j}}(\cdot ; C)(u)=\lim _{j \rightarrow \infty} \int_{C} f\left(x, \frac{x}{\varepsilon_{j}}, \nabla w_{j}(x)\right) d x$;

iii) $w_{j}=u$ on $C \backslash \mathcal{K}_{j}$ for some compact subset $\mathcal{K}_{j} \subset C$ with $\left|C \backslash \mathcal{K}_{j}\right| \rightarrow 0$, for every $j \in \mathbb{N}$.

Proof. The proof relies on De Giorgi's slicing argument introduced in De Giorgi [28]. Let $C \in \mathcal{R}$ and let $\left\{v_{j}\right\} \subset W^{1, p}\left(\Omega ; \mathbb{R}^{d}\right)$ be a sequence given by Lemma 4.2 such that $\left\|u-v_{j}\right\|_{L^{p}(C)} \rightarrow 0$ and

$$
\mathcal{I}^{\left\{\varepsilon_{j}\right\}}(u ; C)=\lim _{j \rightarrow \infty} \mathcal{I}_{\varepsilon_{j}}\left(v_{j} ; C\right) .
$$

Set

$$
\gamma_{0}:=\sup _{j} \int_{C}\left(1+\left|D v_{j}\right|^{p}\right) d x<\infty\left(\text { by }\left(H_{4}\right)\right),
$$

and define for $j \in \mathbb{N}$

$$
K_{j}:=\left|\left[\frac{1}{\left\|v_{j}-u\right\|^{\frac{1}{2}}} L^{p}(C)\right]\right|, \quad M_{j}:=\left|\left[\sqrt{K_{j}}\right]\right|,
$$

and

$$
C_{j}:=\left\{x \in C: \operatorname{dist}(x, \partial C)<\frac{M_{j}}{K_{j}}\right\} .
$$

We observe that by definition $K_{j} \rightarrow \infty$ and $\mathcal{L}^{N}\left(C_{j}\right) \rightarrow 0$ as $j \rightarrow \infty$. For each $j$ subdivide $C_{j}$ into $M_{j}$ disjoint subsets

$$
C_{j}^{i}:=\left\{x \in C_{j}: \operatorname{dist}(x, \partial C) \in\left[\frac{i}{K_{j}}, \frac{i+1}{K_{j}}\right)\right\}, \quad i=0, \ldots, M_{j}-1,
$$

and choose $i_{j} \in\left\{0, \ldots, M_{j}-1\right\}$ such that

$$
\int_{C_{j}^{i_{j}}}\left(1+\left|D v_{j}\right|^{p}\right) d x \leqslant \frac{1}{M_{j}} \int_{C_{j}}\left(1+\left|D v_{j}\right|^{p}\right) d x
$$

for all $i=0, \ldots, M_{j}-1$. Then

$$
\int_{C_{j}^{i_{j}}}\left(1+\left|D v_{j}\right|^{p}\right) d x \leqslant \frac{\gamma_{0}}{M_{j}} .
$$

Let $\phi_{j} \in C_{0}^{\infty}(C)$ be such that $0 \leqslant \phi_{j} \leqslant 1,\left\|D \phi_{j}\right\|_{L^{\infty}} \leqslant K_{j}$,

$$
\phi_{j}:= \begin{cases}1 & \text { if } \operatorname{dist}(x, \partial C) \geqslant \frac{i_{j}+1}{K_{j}}, \\ 0 & \text { if } \operatorname{dist}(x, \partial C) \leqslant \frac{i_{j}}{K_{j}},\end{cases}
$$



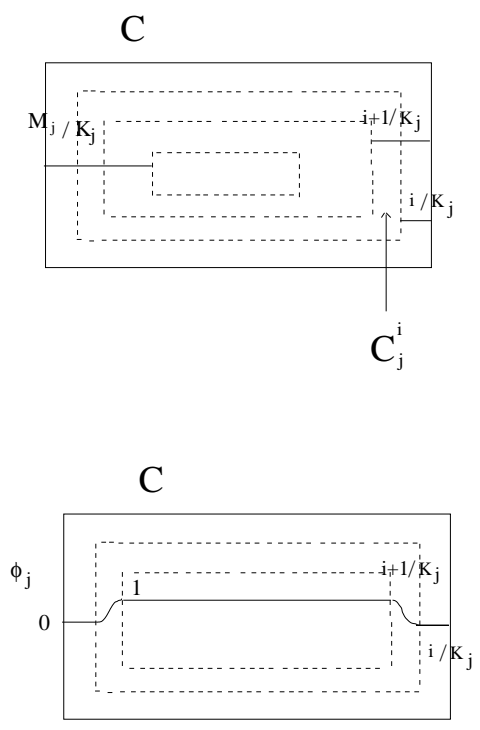

and define $w_{j}:=\phi_{j} v_{j}+\left(1-\phi_{j}\right) u \in W^{1, p}\left(C ; \mathbb{R}^{d}\right)$. Clearly $w_{j} \rightarrow u$ strongly in $L^{p}\left(C ; \mathbb{R}^{d}\right), w_{j}=u$ in $C \backslash \mathcal{K}_{j}$, with $\mathcal{K}_{j}:=\left\{x \in C: \operatorname{dist}(x, \partial C) \geqslant \frac{i_{j}}{K_{j}}\right\}$ and $\left|C \backslash \mathcal{K}_{j}\right| \rightarrow 0$.

Consequently,

$$
\begin{aligned}
\mathcal{I}^{\left\{\varepsilon_{j}\right\}}(u ; C) \geqslant & \limsup _{j \rightarrow \infty} \int_{C} f\left(x, \frac{x}{\varepsilon_{j}}, D w_{j}\right) d x-\beta \limsup _{j \rightarrow \infty} \int_{C_{j} \cap\left\{x: \operatorname{dist}(x, \partial C) \leqslant \frac{i_{j}+1}{K_{j}}\right\}}\left(1+|D u|^{p}\right) d x \\
& \quad-\beta \limsup _{j \rightarrow \infty} \int_{C_{j}^{i_{j}}}\left(1+\left|D v_{j}\right|^{p}\right) d x-\beta \limsup _{j \rightarrow \infty} K_{j}^{p} \int_{C_{j}^{i_{j}}}\left|v_{j}-u\right|^{p} d x \\
\geqslant & \limsup _{j \rightarrow \infty} \int_{C} f\left(x, \frac{x}{\varepsilon_{j}}, D w_{j}\right) d x \\
= & \limsup _{j \rightarrow \infty} \mathcal{I}_{\varepsilon_{j}}\left(w_{j} ; C\right),
\end{aligned}
$$

where we have used (4.4) and the fact that

$$
K_{j}^{p} \int_{C_{j}^{i_{j}}}\left|v_{j}-u\right|^{p} d x \leqslant|| v_{j}-\left.u\right|_{L^{p}\left(C ; \mathbb{R}^{d}\right)} ^{p / 2}
$$

for each $j \in \mathbb{N}$ and $i_{j} \in\left\{0, \ldots, M_{j}-1\right\}$. We conclude that

$$
\Gamma\left(L^{p}(C)\right)-\lim _{j \rightarrow \infty} \mathcal{I}_{\varepsilon_{j}}(\cdot ; C)(u)=\lim _{j \rightarrow \infty} \int_{C} f\left(x, \frac{x}{\varepsilon_{j}}, \nabla w_{j}(x)\right) d x .
$$

Proof of Proposition 4.1. We wish to show that for all $A \in \mathcal{A}(\Omega)$ and $u \in W^{1, p}\left(\Omega, \mathbb{R}^{d}\right)$ 


$$
\begin{aligned}
& \inf \left\{\liminf _{j \rightarrow \infty} \int_{A} f\left(x, \frac{x}{\varepsilon_{j}}, \nabla u_{j}(x)\right) d x: u_{j} \in W^{1, p}\left(A, \mathbb{R}^{d}\right), u_{j} \stackrel{L^{p}\left(A ; \mathbb{R}^{d}\right)}{\longrightarrow} u\right\} \\
= & \inf \left\{\limsup _{j \rightarrow \infty} \int_{A} f\left(x, \frac{x}{\varepsilon_{j}}, \nabla u_{j}(x)\right) d x: u_{j} \in W^{1, p}\left(A, \mathbb{R}^{d}\right), u_{j} \stackrel{L^{p}\left(A ; \mathbb{R}^{d}\right)}{\longrightarrow} u\right\} .
\end{aligned}
$$

Fix $A \in \mathcal{A}(\Omega)$ and $u \in W^{1, p}\left(\Omega, \mathbb{R}^{d}\right)$. To prove (4.5) it suffices to show that for all $\delta>0$ we can find a sequence $\left\{v_{j}\right\}_{j} \subset W^{1, p}\left(A, \mathbb{R}^{d}\right)$ with $v_{j} \stackrel{L^{p}\left(A ; \mathbb{R}^{d}\right)}{\longrightarrow} u$ and such that

$$
\begin{aligned}
& \delta+\inf \left\{\liminf _{j \rightarrow \infty} \int_{A} f\left(x, \frac{x}{\varepsilon_{j}}, \nabla u_{j}(x)\right) d x: u_{j} \in W^{1, p}\left(A, \mathbb{R}^{d}\right), u_{j} \stackrel{L^{p}\left(A ; \mathbb{R}^{d}\right)}{\longrightarrow} u\right\} \\
& \geqslant \limsup _{j \rightarrow \infty} \int_{A} f\left(x, \frac{x}{\varepsilon_{j}}, \nabla v_{j}(x)\right) d x .
\end{aligned}
$$

Choose $\left\{z_{j}\right\}_{j} \subset W^{1, p}\left(A ; \mathbb{R}^{d}\right)$ with $z_{j} \stackrel{L^{p}\left(A ; \mathbb{R}^{d}\right)}{\longrightarrow} u$ such that

$$
\begin{gathered}
\liminf _{j \rightarrow \infty} \int_{A} f\left(x, \frac{x}{\varepsilon_{j}}, \nabla z_{j}(x)\right) d x \\
\leqslant \inf \left\{\liminf _{j \rightarrow \infty} \int_{A} f\left(x, \frac{x}{\varepsilon_{j}}, \nabla u_{j}(x)\right) d x: u_{j} \in W^{1, p}\left(A, \mathbb{R}^{d}\right), u_{j} \stackrel{L^{p}\left(A ; \mathbb{R}^{d}\right)}{\longrightarrow} u\right\}+\frac{\delta}{2}
\end{gathered}
$$

Let $C^{\delta} \in \mathcal{R}$ be such that $\overline{C^{\delta}} \subset A$ and $\mathcal{L}^{N}\left(A \backslash C^{\delta}\right)<<1$, so that

$$
\int_{A \backslash C^{\delta}}\left(1+|\nabla u|^{p}\right) d x \leqslant \frac{\delta}{2 \alpha},
$$

where $\alpha$ is the constant in $\left(H_{4}\right)$. By Lemma 4.3 consider a sequence $\left\{w_{j}^{\delta}\right\}_{j} \in W^{1, p}\left(C^{\delta}, \mathbb{R}^{d}\right)$ such that

$$
\begin{gathered}
w_{j}^{\delta} \stackrel{L^{p}\left(C^{\delta} ; \mathbb{R}^{d}\right)}{\longrightarrow} u, \\
\Gamma\left(L^{p}\left(C^{\delta}\right)\right)-\lim _{j \rightarrow \infty} \mathcal{I}_{\varepsilon_{j}}\left(\cdot ; C^{\delta}\right)(u)=\lim _{j \rightarrow \infty} \int_{C^{\delta}} f\left(x, \frac{x}{\varepsilon_{j}}, \nabla w_{j}^{\delta}(x)\right) d x,
\end{gathered}
$$

and $w_{j}^{\delta}=u$ outside a compact subset $\mathcal{K}_{j}^{\delta}$ of $C^{\delta}$ with $\left|C^{\delta} \backslash \mathcal{K}_{j}^{\delta}\right| \rightarrow 0$ (we could also have used Proposition 11.7 in Braides and Defranceschi [15]). Extend $w_{j}^{\delta}$ by $u$ outside $C^{\delta}$ (still denoted by $w_{j}^{\delta}$ ) so that $w_{j}^{\delta} \stackrel{L^{p}\left(A ; \mathbb{R}^{d}\right)}{\longrightarrow} u$. As $f$ is nonnegative

$$
\begin{aligned}
\liminf _{j \rightarrow \infty} f\left(x, \frac{x}{\varepsilon_{j}}, \nabla z_{j}(x)\right) d x & \geqslant \liminf _{j \rightarrow \infty} \int_{C^{\delta}} f\left(x, \frac{x}{\varepsilon_{j}}, \nabla z_{j}(x)\right) d x \\
& \geqslant \lim _{j \rightarrow \infty} \int_{C^{\delta}} f\left(x, \frac{x}{\varepsilon_{j}}, \nabla w_{j}^{\delta}(x)\right) d x \\
& \geqslant \limsup _{j \rightarrow \infty}\left[\int_{A} f\left(x, \frac{x}{\varepsilon_{j}}, \nabla w_{j}^{\delta}(x)\right) d x-\alpha \int_{A \backslash C^{\delta}}\left(1+|\nabla u|^{p}\right) d x\right] \\
& \geqslant \limsup _{j \rightarrow \infty} \int_{A} f\left(x, \frac{x}{\varepsilon_{j}}, \nabla w_{j}^{\delta}(x)\right) d x-\frac{\delta}{2} .
\end{aligned}
$$


Thus (4.7) and (4.8) yield (4.6).

We now seek to ensure that $\mathcal{I}^{\left\{\varepsilon_{j}\right\}}$, regarded both as a functional on $W^{1, p}\left(\Omega, \mathbb{R}^{d}\right)$ and as a set function, admits an integral representation of the form

$$
\mathcal{I}^{\left\{\varepsilon_{j}\right\}}(u ; A)=\int_{A} g^{\left\{\varepsilon_{j}\right\}}(x, \nabla u(x)) d x .
$$

We will verify that the hypotheses of Theorem 2.10 hold. By Proposition 2.5 the functional $\mathcal{I}^{\left\{\varepsilon_{j}\right\}}(., A)$ is lower semicontinuous with respect to the $L^{p}$ - topology for all $A \in \mathcal{A}(\Omega)$, hence it is sequentially lower semicontinuous with respect to the weak topology in $W^{1, p}$. Thus the only hypothesis that needs to be checked is that $\mathcal{I}^{\left\{\varepsilon_{j}\right\}}(u ;$.$) is a measure.$

Lemma 4.4. For each $u \in W^{1, p}\left(\Omega ; \mathbb{R}^{d}\right), \mathcal{I}^{\left\{\varepsilon_{j}\right\}}(u ;$.$) is the restriction to \mathcal{A}(\Omega)$ of a finite, positive Radon measure.

Proof. Let $u \in W^{1, p}\left(\Omega ; \mathbb{R}^{d}\right)$. In view of Proposition 4.1 let $\left\{u_{j}\right\} \subset W^{1, p}\left(\Omega ; \mathbb{R}^{d}\right)$ be a sequence such that

$$
\mathcal{I}^{\left\{\varepsilon_{j}\right\}}(u ; \Omega)=\lim _{j \rightarrow \infty} \int_{\Omega} f\left(x, \frac{x}{\varepsilon_{j}}, \nabla u_{j}(x)\right) d x,
$$

and consider $\mu_{j}:=f\left(\cdot, \dot{\overline{\varepsilon_{j}}}, \nabla u_{j}\right) \chi_{\Omega}(\cdot) \mathcal{L}^{N}$. By $\left(H_{4}\right)$, and up to a subsequence (still denoted by $\mu_{j}$ ), there exists a finite positive Radon measure on $\mathbb{R}^{N}$ such that

$$
\mu_{j} \stackrel{\star}{\rightarrow} \mu .
$$

We claim that $\mathcal{I}^{\left\{\varepsilon_{j}\right\}}(u ;).\left\lfloor\mathcal{A}(\Omega)=\mu\right.$, that is $\mathcal{I}^{\left\{\varepsilon_{j}\right\}}(u ; A)=\mu(A)$ for all $A \in \mathcal{A}(\Omega)$. We apply Lemma 2.12 with $\Pi(\cdot)=\mathcal{I}^{\left\{\varepsilon_{j}\right\}}(u ;$.$) .$

We start by proving that condition i) in Lemma 2.12 holds, that is $\mathcal{I}^{\left\{\varepsilon_{j}\right\}}(u ;$.$) is nested-subadditive. Given$ $A, B, C \in \mathcal{A}(\Omega)$ with $C \subset \subset B \subset A$ we have to show that

$$
\mathcal{I}^{\left\{\varepsilon_{j}\right\}}(u ; A) \leqslant \mathcal{I}^{\left\{\varepsilon_{j}\right\}}(u ; B)+\mathcal{I}^{\left\{\varepsilon_{j}\right\}}(u ; A \backslash \bar{C}) .
$$

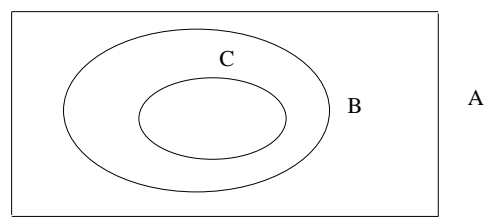

Let $B_{0} \in \mathcal{A}(\Omega), B_{0} \subset B \backslash \bar{C}$, be such that $\mathcal{L}^{N}\left(\partial B_{0}\right)=0$, and for $\delta>0$ choose $C^{\delta}$ and $D^{\delta} \in \mathcal{R}$ with $C^{\delta} \subset B_{0}$ and $D^{\delta} \subset A \backslash \overline{B_{0}}$ such that

$$
\int_{A \backslash\left(C^{\delta} \cup D^{\delta}\right)}\left(1+|\nabla u|^{p}\right) d x<\delta
$$

By Lemma 4.3 there exist two sequences $\left\{v_{j}^{C^{\delta}}\right\}_{j} \subset W^{1, p}\left(C^{\delta} ; \mathbb{R}^{d}\right)$ and $\left\{v_{j}^{D^{\delta}}\right\}_{j} \subset W^{1, p}\left(D^{\delta} ; \mathbb{R}^{d}\right)$ satisfying

$$
\left\|v_{j}^{C^{\delta}}-u\right\|_{L^{p}\left(C^{\delta} ; \mathbb{R}^{d}\right)} \rightarrow 0, \quad\left\|v_{j}^{D^{\delta}}-u\right\|_{L^{p}\left(D^{\delta} ; \mathbb{R}^{d}\right)} \rightarrow 0
$$




$$
\begin{gathered}
\mathcal{I}^{\left\{\varepsilon_{j}\right\}}\left(u ; C^{\delta}\right)=\lim _{j \rightarrow \infty} \mathcal{I}_{\varepsilon_{j}}\left(v_{j}^{C^{\delta}} ; C^{\delta}\right), \quad \mathcal{I}^{\left\{\varepsilon_{j}\right\}}\left(u ; D^{\delta}\right)=\lim _{j \rightarrow \infty} \mathcal{I}_{\varepsilon_{j}}\left(v_{j}^{D^{\delta}} ; D^{\delta}\right), \\
v_{j}^{C^{\delta}}=u \text { on } \partial C^{\delta} \quad \text { and } \quad v_{j}^{D^{\delta}}=u \text { on } \partial D^{\delta} .
\end{gathered}
$$

Extend $v_{j}^{C^{\delta}}$ and $v_{j}^{D^{\delta}}$ by $u$ to all $A$ and set

$$
w_{j}^{\delta}(x):= \begin{cases}v_{j}^{C^{\delta}}(x) & \text { if } x \in B_{0}, \\ v_{j}^{D^{\delta}}(x) & \text { if } x \in A \backslash \overline{B_{0}} .\end{cases}
$$

Clearly $\left\|w_{j}^{\delta}-u\right\|_{L^{p}\left(A ; \mathbb{R}^{d}\right)} \rightarrow 0$ and, if $\alpha$ is the constant in hypothesis $\left(H_{4}\right)$, we have that

$$
\begin{aligned}
\mathcal{I}^{\left\{\varepsilon_{j}\right\}}(u ; A) & \leqslant \liminf _{\delta \rightarrow 0} \liminf _{j \rightarrow \infty} \mathcal{I}_{\varepsilon_{j}}\left(w_{j}^{\delta} ; A\right) \\
& \leqslant \limsup _{\delta \rightarrow 0} \mathcal{I}^{\left\{\varepsilon_{j}\right\}}\left(u ; C^{\delta}\right)+\limsup _{\delta \rightarrow 0} \mathcal{I}^{\left\{\varepsilon_{j}\right\}}\left(u ; D^{\delta}\right)+\alpha \lim _{\delta \rightarrow 0} \int_{A \backslash\left(C^{\delta} \cup D^{\delta}\right)}\left(1+|\nabla u|^{p}\right) d x \\
& \leqslant \mathcal{I}^{\left\{\varepsilon_{j}\right\}}(u ; B)+\mathcal{I}^{\left\{\varepsilon_{j}\right\}}(u ; A \backslash \bar{C}) .
\end{aligned}
$$

To establish condition ii) in Lemma 2.12: Given $A \in \mathcal{A}(\Omega)$ and $\varepsilon>0$, consider $A_{\varepsilon} \in \mathcal{A}(\Omega)$ such that $\overline{A_{\varepsilon}}$ $\subset A$ and

$$
\alpha\left(1+|\nabla u|^{p}\right) \mathcal{L}^{N}\left\lfloor(\Omega)\left(A \backslash \overline{A_{\varepsilon}}\right)<\varepsilon .\right.
$$

Due to the growth conditions $\left(H_{4}\right)$

$$
\begin{aligned}
\mathcal{I}^{\left\{\varepsilon_{j}\right\}}\left(u ; A \backslash \overline{A_{\varepsilon}}\right) & \leqslant \liminf _{j \rightarrow \infty} \int_{A \backslash \overline{A_{\varepsilon}}} f\left(x, \frac{x}{\varepsilon_{j}}, \nabla u(x)\right) d x \\
& \leqslant \alpha \int_{A \backslash \overline{A_{\varepsilon}}}\left(1+|\nabla u(x)|^{p}\right) d x \\
& \leqslant \varepsilon .
\end{aligned}
$$

To show iv) fix $A \in \mathcal{A}(\Omega)$. We have

$$
\begin{aligned}
\mathcal{I}^{\left\{\varepsilon_{j}\right\}}(u ; A) & \leqslant \liminf _{j \rightarrow \infty} \int_{A} f\left(x, \frac{x}{\varepsilon_{j}}, \nabla u_{j}(x)\right) d x \\
& =\liminf _{j \rightarrow \infty} \mu_{j}(A) \\
& \leqslant \mu(\bar{A}) .
\end{aligned}
$$

Finally, to establish iii) take $\Omega^{\prime} \subset \mathbb{R}^{N}$ such that $\Omega \subset \subset \Omega^{\prime}$. As $\left\{\mu_{j}\right\}_{j}$ converges weakly to $\mu$

$$
\mu\left(\Omega^{\prime}\right) \leqslant \lim _{j \rightarrow \infty} \mu_{j}\left(\mathbb{R}^{N}\right)=\lim _{j \rightarrow \infty} \int_{\Omega} f\left(x, \frac{x}{\varepsilon_{j}}, \nabla u_{j}(x)\right) d x=\mathcal{I}^{\left\{\varepsilon_{j}\right\}}(u ; \Omega) .
$$

Therefore

$$
\mu\left(\Omega^{\prime}\right) \leqslant \mathcal{I}^{\left\{\varepsilon_{j}\right\}}(u ; \Omega)
$$


for all such $\Omega^{\prime}$. Hence $\mathcal{I}^{\left\{\varepsilon_{j}\right\}}(u ; \Omega) \geqslant \mu\left(\mathbb{R}^{N}\right)$, and as a consequence of Lemma 2.12 we conclude that $\mathcal{I}^{\left\{\varepsilon_{j}\right\}}(u ; A)=\mu(A)$ for all $A \in \mathcal{A}(\Omega)$.

As a consequence of the integral representation Theorem 2.10 and Remark 2.11 we derive an integral representation formula for $\mathcal{I}^{\left\{\varepsilon_{j}\right\}}$.

Lemma 4.5. There exist a Carathéodory function

$$
g^{\left\{\varepsilon_{j}\right\}}: \Omega \times \mathbb{R}^{d \times N} \rightarrow[0, \infty)
$$

quasiconvex with respect to its second variable for a.e. $x \in \Omega$, satisfying similar growth conditions to those of $f$, and such that

$$
\mathcal{I}^{\left\{\varepsilon_{j}\right\}}(u, A)=\int_{A} g^{\left\{\varepsilon_{j}\right\}}(x, \nabla u(x)) d x
$$

for all $u \in W^{1, p}\left(\Omega ; \mathbb{R}^{d}\right)$ and $A \in \mathcal{A}(\Omega)$.

The remaining of this section is devoted to showing that

$$
g^{\left\{\varepsilon_{j}\right\}}(x, \xi)=f_{\text {hom }}(x, \xi)
$$

for a.e. $x \in \Omega$ and for all $\xi \in \mathbb{R}^{d \times N}$. Let $T \in \mathbb{N}$ and let $\mathcal{S}_{T}$ denote a countable subset of $C_{c}^{\infty}\left((0, T)^{N} ; \mathbb{R}^{d}\right)$ dense in $W_{0}^{1, p}\left((0, T)^{N} ; \mathbb{R}^{d}\right)$. Let $L$ be the set of Lebesgue points $x_{0}$ for all functions

$$
g^{\left\{\varepsilon_{j}\right\}}(\cdot, \eta)
$$

and

$$
x \rightarrow \int_{Q} f(x, T y, \eta+\nabla \phi(T y)) d y
$$

with $\phi \in \mathcal{S}_{T}, \eta \in \mathbb{Q}^{d \times N}$ and $T \in \mathbb{N}$. We have $|\Omega \backslash L|=0$.

Proposition 4.6. $g^{\left\{\varepsilon_{j}\right\}}\left(x_{0}, \xi\right)=f_{\text {hom }}\left(x_{0}, \xi\right)$ for all $x_{0} \in L$ and $\xi \in \mathbb{Q}^{d \times N}$.

Proof. Consider $x_{0} \in L$ and $\xi \in \mathbb{Q}^{d \times N}$. We denote by $Q\left(x_{0}, \delta\right)$ the cube in $\mathbb{R}^{N}$ centered at $x_{0}$ and of radius $\delta>0$. By (4.9) we have

$$
\begin{aligned}
g^{\left\{\varepsilon_{j}\right\}}\left(x_{0}, \xi\right) & =\lim _{\delta \rightarrow 0} \frac{1}{\delta^{N}} \int_{Q\left(x_{0}, \delta\right)} g^{\left\{\varepsilon_{j}\right\}}(x, \xi) d x \\
& =\lim _{\delta \rightarrow 0} \frac{\mathcal{I}^{\left\{\varepsilon_{j}\right\}}\left(\xi \cdot ; Q\left(x_{0}, \delta\right)\right)}{\delta^{N}}
\end{aligned}
$$

Step 1. We first establish the upper bound inequality for the $\Gamma$-limit of $\left\{\mathcal{I}_{\varepsilon_{j}}\right\}$, i.e.

$$
g^{\left\{\varepsilon_{j}\right\}}\left(x_{0}, \xi\right) \leqslant f_{\text {hom }}\left(x_{0}, \xi\right) .
$$

Given $n \in \mathbb{N}$, by Lemma 3.3 let $T_{n} \in \mathbb{N}$ and $\bar{\phi}_{n} \in W_{0}^{1, p}\left(\left(0, T_{n}\right)^{N} ; \mathbb{R}^{d}\right)$ be such that

$$
f_{\mathrm{hom}}\left(x_{0}, \xi\right)+\frac{1}{2 n} \geqslant \frac{1}{T_{n}^{N}} \int_{\left(0, T_{n}\right)^{N}} f\left(x_{0}, y, \xi+\nabla \bar{\phi}_{n}(y)\right) d y .
$$

By conditions $\left(H_{1}\right)$ and $\left(H_{4}\right)$ and by the density of $\mathcal{S}_{T_{n}}$ in $W_{0}^{1, p}\left(\left(0, T_{n}\right)^{N} ; \mathbb{R}^{d}\right)$ we may take $\phi_{n} \in \mathcal{S}_{T_{n}}$ with 


$$
f_{\text {hom }}\left(x_{0}, \xi\right)+\frac{1}{n} \geqslant \frac{1}{T_{n}^{N}} \int_{\left(0, T_{n}\right)^{N}} f\left(x_{0}, y, \xi+\nabla \phi_{n}(y)\right) d y .
$$

Extend $\phi_{n}$ periodically with period $T_{n}$ to $\mathbb{R}^{N}$ (still denoted by $\phi_{n}$ ). For $x \in \mathbb{R}^{N}$ define

$$
u_{j}^{n}(x):=\xi \cdot x+\varepsilon_{j} \phi_{n}\left(\frac{x}{\varepsilon_{j}}\right)
$$

and let $\delta>0$ be small enough so that $\overline{Q\left(x_{0} ; \delta\right)} \subset \Omega$. For fixed $n$ we have $\lim _{j \rightarrow \infty} u_{j}^{n}=v$ in $L^{p}\left(Q\left(x_{0} ; \delta\right) ; \mathbb{R}^{d}\right)$ where $v(x)=\xi \cdot x$. Hence by (4.11)

$$
g^{\left\{\varepsilon_{j}\right\}}\left(x_{0}, \xi\right) \leqslant \liminf _{\delta \rightarrow 0} \liminf _{j \rightarrow \infty} \frac{1}{\delta^{N}} \int_{Q\left(x_{0} ; \delta\right)} f\left(x, \frac{x}{\varepsilon_{j}}, \xi+\nabla \phi_{n}\left(\frac{x}{\varepsilon_{j}}\right)\right) d x .
$$

Define now $h_{n}(y, x):=f\left(x, T_{n} y, \xi+\nabla \phi_{n}\left(T_{n} y\right)\right)$ for all $x \in \Omega$ and a.e. $y \in \mathbb{R}^{N}$, and for all $n \in \mathbb{N}$. By hypotheses $\left(H_{1}\right)-\left(H_{4}\right)$ we have $h_{n} \in L_{\mathrm{per}}^{1}\left(Q ; C\left(\overline{Q\left(x_{0} ; \delta\right)}\right)\right)$ for $n \in \mathbb{N}$, and so by Lemma 2.15

$$
\begin{aligned}
\lim _{j \rightarrow \infty} \int_{Q\left(x_{0} ; \delta\right)} f\left(x, \frac{x}{\varepsilon_{j}}, \xi+\nabla \phi_{n}\left(\frac{x}{\varepsilon_{j}}\right)\right) d x & =\lim _{j \rightarrow \infty} \int_{Q\left(x_{0} ; \delta\right)} h_{n}\left(\frac{x}{T_{n} \varepsilon_{j}}, x\right) d x \\
& =\int_{Q\left(x_{0} ; \delta\right)} \int_{Q} h_{n}(y, x) d y d x \\
& =\int_{Q\left(x_{0} ; \delta\right)} \int_{Q} f\left(x, T_{n} y, \xi+\nabla \phi_{n}\left(T_{n} y\right)\right) d y d x
\end{aligned}
$$

Therefore by identities (4.10), (4.12) and (4.13)

$$
\begin{aligned}
g^{\left\{\varepsilon_{j}\right\}}\left(x_{0}, \xi\right) & \leqslant \liminf _{\delta \rightarrow 0} \frac{1}{\delta^{N}} \int_{Q\left(x_{0} ; \delta\right)} \int_{Q} f\left(x, T_{n} y, \xi+\nabla \phi_{n}\left(T_{n} y\right)\right) d y d x \\
& =\int_{Q} f\left(x_{0}, T_{n} y, \xi+\nabla \phi_{n}\left(T_{n} y\right)\right) d y \\
& =\frac{1}{T_{n}{ }^{N}} \int_{\left(0, T_{n}\right)^{N}} f\left(x_{0}, y, \xi+\nabla \phi_{n}(y)\right) d y \\
& \leqslant f_{\text {hom }}\left(x_{0}, \xi\right)+\frac{1}{n} .
\end{aligned}
$$

Letting $n \rightarrow \infty$ we deduce that

$$
g^{\left\{\varepsilon_{j}\right\}}\left(x_{0}, \xi\right) \leqslant f_{\text {hom }}\left(x_{0}, \xi\right) .
$$

Step 2. We now show that the converse inequality holds, that is

$$
g^{\left\{\varepsilon_{j}\right\}}\left(x_{0}, \xi\right) \geqslant f_{\text {hom }}\left(x_{0}, \xi\right) .
$$

Fix $\delta>0$ small enough so that $\overline{Q\left(x_{0} ; \delta\right)} \subset \Omega$, and consider $\left\{u_{j}^{\delta}\right\}_{j} \subset W^{1, p}\left(Q\left(x_{0} ; \delta\right) ; \mathbb{R}^{d}\right)$ with $\lim _{j \rightarrow \infty} u_{j}^{\delta}=0$ in $L^{p}\left(Q\left(x_{0} ; \delta\right) ; \mathbb{R}^{d}\right)$ and

$$
\mathcal{I}^{\left\{\varepsilon_{j}\right\}}\left(\xi ; Q\left(x_{0} ; \delta\right)\right)=\lim _{j \rightarrow \infty} \int_{Q\left(x_{0} ; \delta\right)} f\left(x, \frac{x}{\varepsilon_{j}}, \xi+\nabla u_{j}^{\delta}(x)\right) d x .
$$


By (4.11)

$$
\begin{aligned}
g^{\left\{\varepsilon_{j}\right\}}\left(x_{0}, \xi\right) & =\lim _{\delta \rightarrow 0} \lim _{j \rightarrow \infty} \frac{1}{\delta^{N}} \int_{Q\left(x_{0} ; \delta\right)} f\left(x, \frac{x}{\varepsilon_{j}}, \xi+\nabla u_{j}^{\delta}(x)\right) d x \\
& =\lim _{\delta \rightarrow 0} \frac{1}{\delta^{N}} \int_{Q\left(x_{0} ; \delta\right)} f\left(x, \frac{x}{\varepsilon_{j(\delta)}}, \xi+\nabla u_{j(\delta)}^{\delta}(x)\right) d x \\
& =\lim _{\delta \rightarrow 0} \int_{Q} f\left(x_{0}+\delta y, \frac{x_{0}+\delta y}{\varepsilon_{j(\delta)}}, \xi+\nabla v_{j(\delta)}^{\delta}(y)\right) d y
\end{aligned}
$$

where $v_{j(\delta)}^{\delta}(y):=\frac{1}{\delta} u_{j(\delta)}^{\delta}\left(x_{0}+\delta y\right) \in W^{1, p}\left(Q ; \mathbb{R}^{d}\right)$, and where using a diagonalization argument we choose the sequence $\{j(\delta)\}_{\delta}$ in such a way that

$$
\frac{\delta}{\varepsilon_{j(\delta)}}>\frac{1}{\delta} \quad \text { and } \quad \lim _{\delta \rightarrow 0}\left\|v_{j(\delta)}^{\delta}\right\|_{L^{p}\left(Q ; \mathbb{R}^{d}\right)}=0
$$

For simplicity denote $j(\delta) \equiv \delta, v_{j(\delta)}^{\delta} \equiv v_{\delta}$ and $u_{j(\delta)}^{\delta} \equiv u_{\delta}$. In view of the coercivity hypothesis $\left(H_{4}\right)$, by the Decomposition Lemma (Theorem 2.20) there exists a subsequence of $\left\{v_{\delta}\right\}_{\delta}$ (still denoted by $\left\{v_{\delta}\right\}_{\delta}$ ) and a sequence $\left\{w_{\delta}\right\}_{\delta} \subset W^{1, \infty}\left(\mathbb{R}^{N} ; \mathbb{R}^{d}\right)$ such that

$$
w_{\delta} \rightarrow 0 \text { in } W^{1, p}, \quad w_{\delta}=0 \text { in a neighborhood of } \partial Q,
$$

$$
\left\{\left|\nabla w_{\delta}\right|^{p}\right\}_{\delta} \text { is equi-integrable }
$$

and

$$
\left|\left\{y \in Q: v_{\delta}(y) \neq w_{\delta}(y)\right\}\right| \rightarrow 0 .
$$

As $f$ is nonnegative, we have

$$
\begin{aligned}
g^{\left\{\varepsilon_{j}\right\}}\left(x_{0}, \xi\right) & \geqslant \limsup _{\delta \rightarrow 0} \int_{\left\{y \in Q: v_{\delta}(y)=w_{\delta}(y)\right\}} f\left(x_{0}+\delta y, \frac{x_{0}+\delta y}{\varepsilon_{\delta}}, \xi+\nabla w_{\delta}(y)\right) d y \\
& =\limsup _{\delta \rightarrow 0} \int_{Q} f\left(x_{0}+\delta y, \frac{x_{0}+\delta y}{\varepsilon_{\delta}}, \xi+\nabla w_{\delta}(y)\right) d y
\end{aligned}
$$

from (4.16), (4.17) and $\left(H_{4}\right)$. Write

$$
\frac{x_{0}}{\varepsilon_{\delta}}:=m_{\delta}+s_{\delta}
$$

with $m_{\delta} \in \mathbb{Z}^{N}$ and $s_{\delta} \in[0,1)^{N}$, and define

$$
x_{\delta}:=\frac{-\varepsilon_{\delta}}{\delta} s_{\delta} .
$$

Note that by (4.15) $x_{\delta} \rightarrow 0$ as $\delta \rightarrow 0$. After changing variables, by the periodicity hypothesis $\left(H_{3}\right)$ and the fact that $\frac{x_{0}+\delta x_{\delta}}{\varepsilon_{\delta}}=m_{\delta} \in \mathbb{Z}^{N}$, we obtain 


$$
\begin{aligned}
& \int_{Q} f\left(x_{0}+\delta y, \frac{x_{0}+\delta y}{\varepsilon_{\delta}}, \xi+\nabla w_{\delta}(y)\right) d y \\
& =\int_{Q-\left\{x_{\delta}\right\}} f\left(x_{0}+\delta\left(y+x_{\delta}\right), \frac{x_{0}+\delta\left(y+x_{\delta}\right)}{\varepsilon_{\delta}}, \xi+\nabla w_{\delta}\left(y+x_{\delta}\right)\right) d y \\
& =\int_{Q-\left\{x_{\delta}\right\}} f\left(x_{0}+\delta\left(y+x_{\delta}\right), \frac{\delta}{\varepsilon_{\delta}} y, \xi+\nabla w_{\delta}\left(y+x_{\delta}\right)\right) d y .
\end{aligned}
$$

Since

$$
\begin{aligned}
\int_{Q \backslash\left(Q-\left\{x_{\delta}\right\}\right)} f\left(x_{0}+\delta\left(y+x_{\delta}\right), \frac{\delta}{\varepsilon_{\delta}} y, \xi+\nabla w_{\delta}\left(y+x_{\delta}\right)\right) d y & \leqslant \beta \int_{Q \backslash\left(Q-\left\{x_{\delta}\right\}\right)}\left(1+\left|\nabla w_{\delta}\left(y+x_{\delta}\right)\right|^{p}\right) d y \\
& =\beta \int_{\left(Q+\left\{x_{\delta}\right\}\right) \backslash Q}\left(1+\left|\nabla w_{\delta}(z)\right|^{p}\right) d z \rightarrow 0
\end{aligned}
$$

as $\delta \rightarrow 0$ (once again because $\left\{\left|\nabla w_{\delta}\right|^{p}\right\}_{\delta}$ is equi-integrable and $\left|\left(Q+\left\{x_{\delta}\right\}\right) \backslash Q\right| \rightarrow 0$ as $\delta \rightarrow 0$ ), we get

$$
g^{\left\{\varepsilon_{j}\right\}}\left(x_{0}, \xi\right) \geqslant \liminf _{\delta \rightarrow 0} \int_{Q} f\left(x_{0}+\delta\left(y+x_{\delta}\right), \frac{\delta}{\varepsilon_{\delta}} y, \xi+\nabla w_{\delta}\left(y+x_{\delta}\right)\right) d y .
$$

Let $a_{i} \in \mathbb{Z}^{N}, i \in\left\{1, \ldots,\left[T_{\delta}\right]^{N}\right\}\left(T_{\delta}:=\delta / \varepsilon_{\delta}\right)$ be such that

$$
\bigcup_{i=1}^{\left[T_{\delta}\right]^{N}}\left(a_{i}+Q\right) \subseteq \frac{\delta}{\varepsilon_{\delta}} Q
$$

and the cubes $a_{i}+Q$ are mutually disjoint. Changing variables, and due to the periodicity hypothesis $\left(H_{3}\right)$, we have

$$
\begin{aligned}
& \int_{Q} f\left(x_{0}+\delta\left(y+x_{\delta}\right), \frac{\delta}{\varepsilon_{\delta}} y, \xi+\nabla w_{\delta}\left(y+x_{\delta}\right)\right) d y \\
& =\left(\frac{\varepsilon_{\delta}}{\delta}\right)^{N} \int_{\frac{\delta}{\varepsilon_{\delta}} Q} f\left(x_{0}+\varepsilon_{\delta} z+\delta x_{\delta}, z, \xi+\nabla w_{\delta}\left(\frac{\varepsilon_{\delta}}{\delta} z+x_{\delta}\right)\right) d z \\
& \geqslant\left(\frac{\varepsilon_{\delta}}{\delta}\right)^{N} \sum_{i=1}^{\left[T_{\delta}\right]^{N}} \int_{a_{i}+Q} f\left(x_{0}+\varepsilon_{\delta} z+\delta x_{\delta}, z, \xi+\nabla w_{\delta}\left(\frac{\varepsilon_{\delta}}{\delta} z+x_{\delta}\right)\right) d z \\
& =\left(\frac{\varepsilon_{\delta}}{\delta}\right)^{N} \sum_{i=1}^{\left[T_{\delta}\right]^{N}} \int_{Q} f\left(x_{0}+\varepsilon_{\delta}\left(z+a_{i}\right)+\delta x_{\delta}, z, \xi+\nabla w_{\delta}\left(\frac{\varepsilon_{\delta}}{\delta}\left(z+a_{i}\right)+x_{\delta}\right)\right) d z .
\end{aligned}
$$

Let $r>0$ be such that $\overline{Q\left(x_{0}, r\right)} \subset \Omega$ and

$$
\varepsilon_{\delta}\left(1+\max _{i}\left\{\left|a_{i}\right|\right\}\right)+\delta\left|x_{\delta}\right|<r
$$


for $\delta$ small enough; note that here we use the fact that $\varepsilon_{\delta}\left|a_{i}\right|=O\left(\varepsilon_{\delta} T_{\delta}\right)=O(\delta)$. Let $\theta_{r} \in C_{0}^{\infty}\left(\mathbb{R}^{N}\right)$ be such that $\theta_{r}=1$ in $Q\left(x_{0}, r\right)$ and $\theta_{r}=0$ on $\mathbb{R}^{N} \backslash Q\left(x_{0}, 2 r\right)$. Define $f_{r}:=f \theta_{r}$.

By the Scorza-Dragoni Theorem (Theorem 2.8) applied to $f_{r}: \mathbb{R}^{N} \times Q \times \mathbb{R}^{d \times N} \rightarrow \mathbb{R}$, given $m \in \mathbb{N}$ let $K_{m}$ be a compact subset of $Q$ such that $\left|Q \backslash K_{m}\right| \leqslant \frac{1}{m}$ and such that $f_{r}: \mathbb{R}^{N} \times K_{m} \times \mathbb{R}^{d \times N} \rightarrow \mathbb{R}$ is continuous. Define

$$
Q_{m, i, \delta}:=\left\{z \in K_{m}: \quad\left|\nabla w_{\delta}\right|\left(\frac{\varepsilon_{\delta}}{\delta}\left(z+a_{i}\right)+x_{\delta}\right) \leqslant m\right\}
$$

for $i \in\left\{1, \ldots,\left[T_{\delta}\right]^{N}\right\}$. Then

$$
\begin{gathered}
\int_{Q} f\left(x_{0}+\varepsilon_{\delta}\left(z+a_{i}\right)+\delta x_{\delta}, z, \xi+\nabla w_{\delta}\left(\frac{\varepsilon_{\delta}}{\delta}\left(z+a_{i}\right)+x_{\delta}\right)\right) d z \\
\geqslant \int_{Q_{m, i, \delta}} f\left(x_{0}+\varepsilon_{\delta}\left(z+a_{i}\right)+\delta x_{\delta}, z, \xi+\nabla w_{\delta}\left(\frac{\varepsilon_{\delta}}{\delta}\left(z+a_{i}\right)+x_{\delta}\right)\right) d z \\
=\int_{Q_{m, i, \delta}} f_{r}\left(x_{0}+\varepsilon_{\delta}\left(z+a_{i}\right)+\delta x_{\delta}, z, \xi+\nabla w_{\delta}\left(\frac{\varepsilon_{\delta}}{\delta}\left(z+a_{i}\right)+x_{\delta}\right)\right)-f_{r}\left(x_{0}, z, \xi+\nabla w_{\delta}\left(\frac{\varepsilon_{\delta}}{\delta}\left(z+a_{i}\right)+x_{\delta}\right)\right) \\
+\int_{Q_{m, i, \delta}} f\left(x_{0}, z, \xi+\nabla w_{\delta}\left(\frac{\varepsilon_{\delta}}{\delta}\left(z+a_{i}\right)+x_{\delta}\right)\right)
\end{gathered}
$$

As $f_{r}: \overline{Q\left(x_{0}, r\right)} \times K_{m} \times \bar{B}(\xi, m) \rightarrow \mathbb{R}$ is uniformly continuous, the first term above goes to zero as $\delta \rightarrow 0$. Thus, from (4.20) and by the periodicity of $f$ we have

$$
\begin{aligned}
g^{\left\{\varepsilon_{j}\right\}}\left(x_{0}, \xi\right) & \geqslant \limsup _{m \rightarrow \infty} \limsup _{\delta \rightarrow 0}\left(\frac{\varepsilon_{\delta}}{\delta}\right)^{N} \sum_{i=1}^{\left[T_{\delta}\right]^{N}} \int_{Q_{m, i, \delta}} f\left(x_{0}, z, \xi+\nabla w_{\delta}\left(\frac{\varepsilon_{\delta}}{\delta}\left(z+a_{i}\right)+x_{\delta}\right)\right) d z \\
& =\limsup _{m \rightarrow \infty} \limsup _{\delta \rightarrow 0} \sum_{i=1}^{\left[T_{\delta}\right]^{N}} \int_{K_{m, i, \delta}} f\left(x_{0}, \frac{\delta}{\varepsilon_{\delta}} y, \xi+\nabla w_{\delta}\left(y+x_{\delta}\right)\right) d y
\end{aligned}
$$

where

$$
K_{m, i, \delta}:=\left\{y \in \frac{\varepsilon_{\delta}}{\delta}\left(K_{m}+a_{i}\right): \quad\left|\nabla w_{\delta}\right|\left(y+x_{\delta}\right) \leqslant m\right\}
$$

Note that

$$
\limsup _{m \rightarrow \infty} \limsup _{\delta \rightarrow 0}\left|Q \backslash \bigcup_{i=1}^{\left[T_{\delta}\right]^{N}} K_{m, i, \delta}\right|=0
$$

because 


$$
\begin{aligned}
\left|Q \backslash \bigcup_{i=1}^{\left[T_{\delta}\right]^{N}} \frac{\varepsilon_{\delta}}{\delta}\left(K_{m}+a_{i}\right)\right| & =\left(\frac{\varepsilon_{\delta}}{\delta}\right)^{N}\left|\frac{\delta}{\varepsilon_{\delta}} Q \backslash \bigcup_{i=1}^{\left[T_{\delta}\right]^{N}}\left(K_{m}+a_{i}\right)\right| \\
& \leqslant\left(\frac{\varepsilon_{\delta}}{\delta}\right)^{N}\left|\frac{\delta}{\varepsilon_{\delta}} Q \backslash \bigcup_{i=1}^{\left[T_{\delta}\right]^{N}}\left(Q+a_{i}\right)\right|+\left(\frac{\varepsilon_{\delta}}{\delta}\right)^{N}\left|\bigcup_{i=1}^{\left[T_{\delta}\right]^{N}}\left(Q+a_{i}\right) \backslash\left(\bigcup_{j=1}^{\left[T_{\delta}\right]^{N}}\left(K_{m}+a_{j}\right)\right)\right| \\
& \leqslant\left(\frac{\varepsilon_{\delta}}{\delta}\right)^{N}\left[\left(\frac{\delta}{\varepsilon_{\delta}}\right)^{N}-\left[T_{\delta}\right]^{N}\right]+\left(\frac{\varepsilon_{\delta}}{\delta}\right)^{N}\left[T_{\delta}\right]^{N}\left|Q \backslash K_{m}\right| \\
& \leqslant\left(\frac{\varepsilon_{\delta}}{\delta}\right)^{N}\left[\left(\frac{\delta}{\varepsilon_{\delta}}\right)^{N}-\left(\frac{\delta}{\varepsilon_{\delta}}-1\right)^{N}\right]+\left(\frac{\varepsilon_{\delta}}{\delta}\right)^{N} \frac{\left[T_{\delta}\right]^{N}}{m} \\
& =\left[1-\left(1-\frac{\varepsilon_{\delta}}{\delta}\right)^{N}\right]+\frac{1}{m} \rightarrow 0
\end{aligned}
$$

as $\delta \rightarrow 0$ and then $m \rightarrow \infty$. In addition we have that

$$
\left\{y \in Q: \quad\left|\nabla w_{\delta}\left(y+x_{\delta}\right)\right| \geqslant m\right\} \leqslant \frac{1}{m} \int_{Q-\left\{x_{\alpha}\right\}}\left|\nabla w_{\delta}\right| d y \rightarrow 0 .
$$

when $\delta \rightarrow 0$ and then $m \rightarrow \infty$. Thus

$$
g^{\left\{\varepsilon_{j}\right\}}\left(x_{0}, \xi\right) \geqslant \liminf _{\delta \rightarrow 0} \int_{Q} f\left(x_{0}, \frac{\delta}{\varepsilon_{\delta}} y, \xi+\nabla w_{\delta}\left(y+x_{\delta}\right)\right) d y .
$$

To compare $g^{\left\{\varepsilon_{j}\right\}}\left(x_{0}, \xi\right)$ with $f_{\text {hom }}\left(x_{0}, \xi\right)$ we need to modify $w_{\delta}\left(\cdot+x_{\alpha}\right)$ close to the boundary of $Q$ in order to be admissible for $f_{\text {hom }}$. For this purpose, define the sets

$$
L_{\delta}:=\left\{y \in Q: \operatorname{dist}(\mathrm{y}, \partial \mathrm{Q}) \leqslant\left|x_{\delta}\right|\right\}, \quad M_{\delta}=\left\{y \in Q: \operatorname{dist}(\mathrm{y}, \partial \mathrm{Q}) \geqslant 2\left|x_{\delta}\right|\right\},
$$

and

$$
S_{\delta}=\left\{y \in Q: \operatorname{dist}(\mathrm{y}, \partial \mathrm{Q}) \in\left(\left|x_{\delta}\right|, 2\left|x_{\delta}\right|\right)\right\} .
$$

Let $\phi_{\delta} \in C_{c}^{\infty}(Q ; \mathbb{R})$ with $\left\|\nabla \phi_{\delta}\right\|_{\infty} \leqslant \frac{C}{\left|x_{\delta}\right|}$ be such that

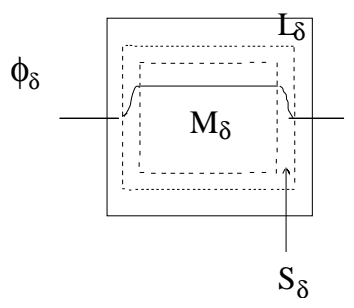

$$
\phi_{\delta}(y)= \begin{cases}1 & \text { if } y \in M_{\delta}, \\ 0 & \text { if } y \in L_{\delta},\end{cases}
$$

and set $\psi_{\delta}(y):=\phi_{\delta}(y) w_{\delta}\left(y+x_{\delta}\right)+\left(1-\phi_{\delta}(y)\right) w_{\delta}(y) \in W_{0}^{1, \infty}\left(Q ; \mathbb{R}^{d}\right)$. The goal is to show that 


$$
g^{\left\{\varepsilon_{j}\right\}}\left(x_{0}, \xi\right) \geqslant \liminf _{\delta \rightarrow 0} \int_{Q} f\left(x_{0}, \frac{\delta}{\varepsilon_{\delta}} y, \xi+\nabla \psi_{\delta}(y)\right) d y
$$

i.e.

$$
g^{\left\{\varepsilon_{j}\right\}}\left(x_{0}, \xi\right) \geqslant \liminf _{\delta \rightarrow 0}\left(\frac{\varepsilon_{\delta}}{\delta}\right)^{N} \int_{\frac{\delta}{\varepsilon_{\delta}} Q} f\left(x_{0}, y, \xi+\nabla \zeta_{\delta}(y)\right) d y
$$

with $\zeta_{\delta}(y):=\frac{\delta}{\varepsilon_{\delta}} \psi_{\delta}\left(\frac{\varepsilon_{\delta}}{\delta} y\right) \in W_{0}^{1, \infty}\left(\frac{\delta}{\varepsilon_{\delta}} Q ; \mathbb{R}^{d}\right)$, and, consequently,

$$
g^{\left\{\varepsilon_{j}\right\}}\left(x_{0}, \xi\right) \geqslant f_{\text {hom }}\left(x_{0}, \xi\right) .
$$

To prove (4.22) we observe that

$$
\begin{aligned}
\liminf _{\delta \rightarrow 0} \int_{Q} f\left(x_{0}, \frac{\delta}{\varepsilon_{\delta}} y, \xi+\nabla \psi_{\delta}(y)\right) d y \leqslant & \liminf _{\delta \rightarrow 0} \int_{Q} f\left(x_{0}, \frac{\delta}{\varepsilon_{\delta}} y, \xi+\nabla w_{\delta}\left(y+x_{\delta}\right)\right) d y \\
& +\beta \limsup _{\delta \rightarrow 0} \int_{L_{\delta}}\left(1+\left|\nabla w_{\delta}(x)\right|^{p}\right) d x \\
& +\beta \limsup _{\delta \rightarrow 0} \int_{S_{\delta}}\left(\left|\nabla w_{\delta}\left(x+x_{\delta}\right)\right|^{p}+\left|\nabla w_{\delta}(x)\right|^{p}\right) d x \\
& +\beta \limsup _{\delta \rightarrow 0}\left\|\nabla \phi_{\delta}\right\|_{\infty}^{p} \int_{S_{\delta}}\left|w_{\delta}\left(x+x_{\delta}\right)-w_{\delta}(x)\right|^{p} d x
\end{aligned}
$$

Due to the integrability property of $\left\{\left|\nabla w_{\delta}\right|^{p}\right\}_{\delta}$ we have

$$
\lim _{\delta \rightarrow 0} \int_{L_{\delta}}\left(1+\left|\nabla w_{\delta}(x)\right|^{p}\right) d x=0=\lim _{\delta \rightarrow 0} \int_{S_{\delta}}\left(\left|\nabla w_{\delta}\left(x+x_{\delta}\right)\right|^{p}+\left|\nabla w_{\delta}(x)\right|^{p}\right) d x .
$$

Moreover,

$$
\begin{aligned}
\left\|\nabla \phi_{\delta}\right\|_{\infty}^{p} \int_{S_{\delta}}\left|w_{\delta}\left(x+x_{\delta}\right)-w_{\delta}(x)\right|^{p} d x & \leqslant \frac{\beta}{\left|x_{\delta}\right|^{p}} \int_{S_{\delta}}\left|\int_{0}^{1} \frac{d w_{\delta}\left(x+t x_{\delta}\right)}{d t} d t\right|^{p} d x \\
& \leqslant \frac{\beta}{\left|x_{\delta}\right|^{p}} \int_{S_{\delta}} \int_{0}^{1}\left|\nabla w_{\delta}\left(x+t x_{\delta}\right)\right|^{p} \cdot\left|x_{\delta}\right|^{p} d t d x \\
& =\beta \int_{S_{\delta}} \int_{0}^{1}\left|\nabla w_{\delta}\left(x+t x_{\delta}\right)\right|^{p} d t d x \\
& =\beta \int_{0}^{1} \int_{S_{\delta}+t x_{\delta}}\left|\nabla w_{\delta}(y)\right|^{p} d y d t \\
& \leqslant \beta \int_{N_{\delta}}\left|\nabla w_{\delta}(y)\right|^{p} d y
\end{aligned}
$$

where

$$
N_{\delta}=\left\{x \in Q: \operatorname{dist}(\mathrm{x}, \partial \mathrm{Q}) \leqslant 3\left|x_{\delta}\right|\right\}
$$

and consequently, as above, 


$$
\lim _{\delta \rightarrow 0}\left\|\nabla \phi_{\delta}\right\|_{\infty}^{p} \int_{S_{\delta}}\left|w_{\delta}\left(x+x_{\delta}\right)-w_{\delta}(x)\right|^{p} d x=0 .
$$

Hence (4.22) holds by (4.21) and (4.23)-(4.25).

As a consequence we derive the equality (a.e.) of the density functions $g^{\left\{\varepsilon_{j}\right\}}$ and $f_{\text {hom }}$.

Corollary 4.7. $g^{\left\{\varepsilon_{j}\right\}}(x, \xi)=f_{\text {hom }}(x, \xi)$ for a.e. $x \in \Omega$ and for all $\xi \in \mathbb{R}^{d \times N}$.

Proof. In view of Proposition 4.6 we have that for a.e. $x \in \Omega$ and for all $\xi \in \mathbb{Q}^{d \times N}, g^{\left\{\varepsilon_{j}\right\}}(x ; \xi)=f_{\text {hom }}(x ; \xi)$. Since $g^{\left\{\varepsilon_{j}\right\}}(x ; \cdot)$ and $f_{\text {hom }}(x ; \cdot)$ are continuous, the equality $g^{\left\{\varepsilon_{j}\right\}}(x ; \xi)=f_{\text {hom }}(x ; \xi)$ holds true for all $x \in \Omega$ and for all $\xi \in \mathbb{R}^{d \times N}$.

The proof of our main result is now straightforward.

Proof of Theorem 1.1 As a consequence of Corollary 4.7 the functional $\mathcal{I}_{\text {hom }}$ is well defined and $\Gamma\left(L^{p}(A)\right)$ limit of $\mathcal{I}_{\varepsilon_{j}}(\cdot ; A)$ is equal to $\mathcal{I}_{\text {hom }}(\cdot, A)$. In particular, since it does not depend upon the extracted subsequence, in view of Proposition 2.3 , the whole sequence $\mathcal{I}_{\varepsilon}(\cdot ; A) \Gamma\left(L^{p}(A)\right)$-converges to $\mathcal{I}_{\text {hom }}(\cdot ; A)$. Taking $A=\Omega$ we conclude the proof of Theorem 1.1.

\section{$5 \quad$ A different set of hypotheses}

As mentioned in the Introduction, it is possible assuming that the integrand $f=f(x, y, \xi)$ is measurable in $x$, continuous with respect to the pair $(y, \xi)$, and $Q$-periodic as a function of the variable $y$. Precisely, the following result holds.

Theorem 5.1. Let $f: \Omega \times \mathbb{R}^{N} \times \mathbb{R}^{d \times N} \rightarrow \mathbb{R}$ be a function such that

$\left(H_{1}\right) f(x, \cdot, \cdot)$ is continuous a.e. $x \in \Omega$;

$\left(H_{2}\right) f(\cdot, y, \xi)$ is measurable for all $y \in \mathbb{R}^{N}$ and $\xi \in \mathbb{R}^{d \times N}$;

$\left(H_{3}\right) f(x, \cdot \xi)$ is $Q$-periodic for a.e. $x \in \Omega$ and all $\xi \in \mathbb{R}^{d \times N}$;

$\left(H_{4}\right)$ there exist a real number $p>1$ and a constant $\alpha>0$ such that

$$
\frac{|\xi|^{p}}{\alpha}-\alpha \leqslant f(x, y, \xi) \leqslant \alpha\left(1+|\xi|^{p}\right),
$$

for a.e. $x \in \Omega$, all $y \in \mathbb{R}^{N}$ and $\xi \in \mathbb{R}^{d \times N}$.

For each $\varepsilon>0$ define the functional $\mathcal{I}_{\varepsilon}: L^{p}\left(\Omega ; \mathbb{R}^{d}\right) \rightarrow[0, \infty]$ by

$$
\mathcal{I}_{\varepsilon}(u):= \begin{cases}\int_{\Omega} f\left(x, \frac{x}{\varepsilon}, \nabla u(x)\right) d x & \text { if } u \in W^{1, p}\left(\Omega ; \mathbb{R}^{d}\right), \\ \infty & \text { otherwise. }\end{cases}
$$

If $u \in L^{p}\left(\Omega ; \mathbb{R}^{d}\right)$ then

$$
\mathcal{I}_{\mathrm{hom}}(u):=\Gamma\left(L^{p}(\Omega)\right)-\lim _{\varepsilon \rightarrow 0} \mathcal{I}_{\varepsilon}(u)= \begin{cases}\int_{\Omega} f_{\mathrm{hom}}(x, \nabla u(x)) d x & \text { if } u \in W^{1, p}\left(\Omega ; \mathbb{R}^{d}\right), \\ +\infty & \text { otherwise }\end{cases}
$$


where the integrand $f_{\text {hom }}$ is given by

$$
f_{\text {hom }}(x, \xi):=\lim _{T \rightarrow+\infty} \inf _{\phi}\left\{\frac{1}{T^{N}} \int_{(0, T)^{N}} f(x, y, \xi+\nabla \phi(y)) d y, \phi \in W_{0}^{1, p}\left((0, T)^{N} ; \mathbb{R}^{d}\right)\right\}
$$

for a.e. $x \in \Omega$ and all $\xi \in \mathbb{R}^{d \times N}$. Moreover $f_{\text {hom }}$ is (equivalent to) a Carathéodory function, satisfies similar p-coercivity and p-growth conditions to those of $f$, and $f_{\mathrm{hom}}(x, \cdot)$ is quasiconvex for a.e. $x \in \Omega$.

The argument of the proof follows closely that of the proof of Theorem 1.1, although, it is somewhat less technical. In particular, in (3.27) we need now to ensure that

$$
(y, z) \mapsto \frac{\partial f}{\partial \eta}(x, z, \varphi(y, z)) \in L^{q}\left(Q ; C_{\mathrm{per}}(Q)\right)
$$

and Scorza Dragoni's Theorem in Proposition 4.6 must be used in a different way (see Baía and Fonseca [6] for details).

\section{Appendix}

In this section we recall an auxiliar lemma of use for the proof of Theorems 1.1 and 5.1. First we invoke

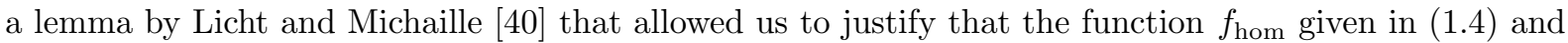
(5.1) is well defined (see Lemma 3.1 above).

Lemma 6.1. Let $N \in \mathbb{N}$ with $N \geqslant 1$ and let $S: \mathcal{A}\left(\mathbb{R}^{N}\right) \rightarrow \mathbb{R}^{+}$be such that

i) $S(A) \leqslant \beta \mathcal{L}^{N}(A)$, for all $A \in \mathcal{A}\left(\mathbb{R}^{N}\right)$, where $\beta$ is a positive constant,

ii) $S(C) \leqslant S(A)+S(B)$ for all $A, B, C \in \mathcal{A}\left(\mathbb{R}^{N}\right)$, with $A \cap B \neq \emptyset, \bar{C}=\bar{A} \cup \bar{B}$,

iii) there exists $T \subset \mathbb{R}^{N}$ and $M>0$ such that $T+[0, M)^{N}=\mathbb{R}^{N}$ and $S(A+\tau)=S(A)$ for all $A \in \mathcal{A}\left(\mathbb{R}^{N}\right)$ and $\tau \in T$.

Then, for any cube $A$ of the form $[a, b)^{N}$ there exists the limit of the sequence $\left\{\frac{S(s A)}{\mathcal{L}^{N}(s A)}\right\}$ as $s \rightarrow \infty$ and

$$
\lim _{s \rightarrow \infty} \frac{S(s A)}{\mathcal{L}^{N}(s A)}=\lim _{s \rightarrow \infty} \frac{S\left([0, s)^{N}\right)}{s^{N}} .
$$

Furthermore, if $\left\{S_{L}\right\}_{L}$ is a family of set functions satisfying $\left.\left.i\right), i i\right)$, iii) for $C, T$ and $M$ independent of $L$, then the above limits are attained uniformly with respect to $L$.

Acknowledgements: M. Baía thanks Alexei Novikov for his interest and for fruitful discussions on the subject of this paper. The research of M. Baía was partially supported by Fundação para a Ciência e a Tecnologia under Grant PRAXIS XXI SFRH $\backslash B D \backslash 1174 \backslash 2000$, Fundo Social Europeu, the Department of Mathematical Sciences of Carnegie Mellon University and its Center for Nonlinear Analysis (CNA). The research of I. Fonseca was partially funded by the National Science Foundation (NSF) under Grants DMS0103799 and DMS-0401763. The authors thank the CNA (NSF Grants DMS-9803791 and DMS-0405343) for its support during the preparation of this work. 


\section{References}

[1] E. Acerbi and N. Fusco, Semicontinuity problems in the calculus of variations, Arch. Rational Mech. Anal. 86 (1984), 125-145.

[2] G. Allaire, Homogenization and two-scale convergence, SIAM J. Math. Anal. 23 (1992), No. 6, $1482-1518$.

[3] G. Allaire and M. Briane, Multiscale convergence and reiterated homogenisation, Proc. Roy. Soc. Edinburgh Sect. A 126 (1996), No. 2, 297-342.

[4] R. De Arcangelis and G. Gargiulo, Homogenization of integral functionals with linear growth defined on vector-valued functions, No. DEA 2 (1995), 371-416.

[5] J.-F. Babadjian and M. Baía, Multiscale nonconvex relaxation and application to thin films, to appear in Asymptotic Analysis.

[6] M. Baía and I. Fonseca, $\Gamma$-convergence of functionals with periodic integrands via 2-scale convergence, CNA Research Report No. CNA-010-05, 2005.

[7] J. M. Ball and F. Murat, $W^{1, p}$-quasiconvexity and variational problems for multiples integrals, J. Funct. Anal. 58 (1984), 225-253.

[8] M. Barchiesi, Multiscale homogenization of convex functionals with discontinuous integrands, to appear in Journal of Convex Analysis.

[9] A. Bensoussan, J. L. Lions and G. Papanicolau, Asymptotic Analysis for Periodic Sructures, NorthHolland, Amsterdam, 1978.

[10] L. Berlyand, D. Cioranescu and D. Golovaty, Homogenization of a Ginzburg-Landau model with a surface term for a liquid crystalline medium with inclusions, Journal des Mathématiques Pures et Appliquées 84 (2004), No. 1, 97-136.

[11] A. Braides, Homogenization of noncoercive integrals, (Italian) Ricerche Mat. 32 (1983), No. 2, 347368.

[12] A. Braides, Homogenization of some almost periodic coercive functional, Rend. Accad. Naz. Sci. XL 103 (1985), 313-322.

[13] A. Braides, Г-convergence for Beginners, Oxford Lecture Series in Mathematics and its Applications, Oxford University Press, Oxford, 2002.

[14] A. Braides and V. Chiadò Piat, A derivation formula for convex integral functions defined on $B V(\Omega)$, J. Convex Anal. 2 (1995), 69-85.

[15] A. Braides and A. Defranceschi, Homogenization of Multiple Integrals, Oxford Lecture Series in Mathematics and its Applications, The Clarendon Press, Oxford University Press, New York, 1998.

[16] A. Braides, F. Fonseca and G. Francfort, 3D-2D asymptotic analysis for inhomogeneous thin films, Indiana Univ. Math. J. 49 (2000), No. 4, 1367-1404.

[17] A. Braides and D. Lukkassen, Reiterated homogenization of integral functionals, Math. Models Methods Appl. Sci. 10 (2000), No. 1, 47-71.

[18] G. Bouchitté, Homogénéisation sur $B V(\Omega)$ de fonctionnelles intégrales à croissance linéaire. Application à um problème d'analyse limite en plasticité, C. R. Acad. Sci. Paris Sér. I. Math. 301 (1995), $785-788$. 
[19] G. Bouchitté, F. Fonseca and L. Mascarenhas, A global method for relaxation, Arch. Rational Mech. Anal. 145 (1998), 51-98.

[20] G. Buttazzo and G. Dal Maso, Integral representation and relaxation of local functional, Nonlinear Analysis, Theory, Methods \& Applications 9 (1985), No. 6, 515-532.

[21] L. Carbone and C. Sbordone, Some properties of $\Gamma$-limits of integral functionals, Ann. Mat. Pura Appl. (4) 122 (1979), 1-60.

[22] L. Carbone, D. Cioranescu, R. De Arcangelis and A. Gaudiello, Homogenization of unbounded functionals and nonlinear elastomers. The case of the fixed constraints set., ESAIM Control Optim. Calc. Var. 10 (2004), No. 1, 53-83 (electronic).

[23] D. Cioranescu, A. Damlamian and R. De Arcangelis, Homogenization of nonlinear integrals via the periodic unfolding method, C. R. Math. Acad. Sci. Paris 339 (2004), No. 1, 77-82.

[24] B. Dacorogna, Direct Methods in the Calculus of Variations, Springer-Verlag, 1989.

[25] G. Dal Maso, An introduction to $\Gamma$ - convergence, Progress in Nonlinear Differential Equations and their Applications, Birkhäuser Boston, Inc., Boston, MA, 1993.

[26] G. Dal Maso, I. Fonseca, G. Leoni and M. Morini, Higher order quasiconvexity reduces to quasiconvexity, Arch. Rational Mech. Anal. 171 (2004) 55-81.

[27] E. De Giorgi and G. Dal Maso, Г-convergence and Calculus of variations, Mathematical theories of optimization (Genova 1981), 121-143, Lecture Notes in Math. 979, Springer, Berlin, 1983.

[28] E. De Giorgi, Sulla convergenza di alcune successioni d'integrali del tipo dell'area, Rend. Mat. (6) 8 (1975), 277-294.

[29] E. De Giorgi and T. Franzoni, Su un tipo di convergenza variazionale, Atti Accad. Naz. Lincei Rend. Cl. Sci. Fis. Mat. Natur. (8) 58 (1975), No. 6, 842-850.

[30] E. De Giorgi and G. Letta, Une notion générale de convergence faible pour des fonctions croissantes d'emsemble, Ann. Sc. Norm. Sup. Pisa Cl. Sci (4) 4 (1977), 61-99.

[31] E. De Giorgi and S. Spagnolo, Sulla convergenza degli integrali dell'energia per operatori ellittici del secondo ordine, Boll. Un. Mat. Ital. (4) 8 (1973), 391-411.

[32] P. Donato, Some remarks on the weak convergence of functions oscillating nonuniformly [Ricerche Mat. 32 (1983), no. 2, 203-219], (Italian) Ricerche Mat. 33 (1984), No. 1, 144.

[33] I. Ekeland, R. Temam, Analyse Convexe et Problèmes Variationnels, Dunod, Gauthiers-Villars, Paris, 1974.

[34] L. C. Evans, R. F. Gariepy, Measure Theory and Fine Properties of Functions, Boca Raton, CRC Press, 1992.

[35] I. Fonseca and G. Leoni, Modern Methods in the Calculus of Variations in $L^{p}$ Spaces, in preparation.

[36] I. Fonseca and J. Malý, Relaxation of multiple integrals in Sobolev spaces below the growth exponent for the energy density, Ann. Inst. H. Poincaré, Anal. Non Linéaire 14 (1997), No.3, 309-338.

[37] I. Fonseca, S. Müller and P. Pedregal, Analysis of concentration and oscillations effects generated by gradients, SIAM J. Math. Anal 29 (1998), No. 3, 736-756. 
[38] I. Fonseca and E. Zappale, Multiscale relaxation of convex functionals, J. Convex Anal. 10 (2003), No. 2, 325-350.

[39] V. V. Jikov, S. M. Kozlov and O. A. Oleinik, Homogenization of Differential Operators and Integral Functionals, Springer-Verlag, 1996.

[40] C. Licht and G. Michaille, Global-local subadditive ergodic theorems and application to homogenization in elasticity, Ann. Math. Blaise Pascal 9 (2002), No. 1, 21-62.

[41] D. Lukkassen, Formulae and bounds connected to optimal design and homogenization of partial differential operators and integrals functionals, Ph. D. Thesis Department of Mathematics, Tromöm University, Norway, 1996.

[42] D. Lukkassen, G. Nguetseng and P. Wall, Two-scale convergence, Int. J. Pure Appl. Math. 2 (2002), no. $1,35-86$.

[43] J. Kristensen, Finite functionals and Young measures generated by gradients of Sobolev functions, Mat-Report 1994-34, Mathematical Institute, Technical University of Denmark, 1994.

[44] P. Marcellini, Periodic solutions and homogenization of nonlinear variational problems, Annali Mat. Pura Appl. 117 (1978), 139-152.

[45] P. Marcellini, Approximation of quasiconvex functions, and lower semicontinuity of multiple integrals, Manuscripta Math. 51 (1985), No. 1-3, 1-28.

[46] C. B. Morrey, Quasi-convexity and the semicontinuity of multiple integrals, Pacif. J. Math. 2 (1952), $25-53$.

[47] S. Müller, Homogenization of nonconvex integral functionals and cellular elastic materials, Arch. Rational Mech. Anal. 99 (1987), 189-212.

[48] F. Murat, Séminaire d'Analyse Fonctionnele et Numérique, 1977/78, Univ. d'Alger.

[49] G. Nguetseng, A general convergence result for a functional related to the theory of homogenization, SIAM J. Math. Anal. 20 (1989), No. 3, 608-623.

[50] G. Nguetseng, Asymptotic analysis for a stiff variational problem arising in mechanics, SIAM J. Math. Anal. 21 (1990), No. 6, 1394-1414.

[51] P. Pedregal, Multiscale Young measures, Trans. Am. Math. Soc. (2005) (electronic).

[52] E. Sanchez-Palencia, Nonhomogeneous media and vibration theory, Lecture Notes in Phys. 127 Springer-Verlag, New York, 1980.

[53] S. Spagnolo, Sulla convergenza di soluzioni di equazioni paraboliche ed ellitiche, Ann. Scuola Norm. Sup. Pisa 22 (1968), 577-597.

[54] L. Tartar, Cours Peccot au Collègge de France. 
Margarida Baía

Departmento de Matemática

Instituto Superior Técnico

Av. Rovisco Pais, 1049-001 Lisboa, Portugal

E-mail: mbaia@math.ist.utl.pt

Irene Fonseca

Center for Nonlinear Analysis

Department of Mathematical Sciences

Carnegie Mellon University

Pittsburgh, PA 15213-3890

E-mail: fonseca@andrew.cmu.edu 